\title{
Relación entre descargas y citas de revistas científicas en el ámbito de la documentación: el caso de las universidades públicas de Castilla y León
}

\author{
Blanca Rodríguez-Bravo*, Andrés Fernández-Ramos*, Críspulo Travieso-Rodríguez** \\ * Área de Biblioteconomía y Documentación, Facultad de Filosofía y Letras, Universidad de León. \\ Correo-e: blanca.rodriguez@unileon.es | ORCID iD: https://orcid.org/0000-0002-9476-7602 \\ Correo-e: afernr@unileon.es | ORCID iD: https://orcid.org/0000-0002-6869-602X \\ **Dpto. Biblioteconomía y Documentación, Universidad de Salamanca \\ Correo-e: ctravieso@usal.es | ORCID iD: https://orcid.org/0000-0002-0774-0728
}

Recibido: 15-06-20; 2a versión: 13-10-20; Aceptado: 19-10-20; Publicado: 04-10-21

Cómo citar este artículo/Citation: Rodríguez-Bravo, B.; Fernández-Ramos, A.; Travieso-Rodríguez, C. (2021). Relación entre descargas y citas de revistas científicas en el ámbito de la documentación: el caso de las universidades públicas de Castilla y León. Revista Española de Documentación Científica, 44 (4), e307. https://doi.org/ 10.3989/redc.2021.4.1806

\begin{abstract}
Resumen: Se indaga en la utilidad de las revistas electrónicas de los big deals contratados por las universidades públicas de la Comunidad Autónoma de Castilla y León (Universidad de Burgos, Universidad de León, Universidad de Salamanca y Universidad de Valladolid) a partir del análisis de las descargas y citas realizadas por sus investigadores. El análisis se circunscribe a la producción científica presente en Scopus en la categoría Library \& Information Science (LIS) durante el periodo 2011-2017 y a las estadísticas de descargas que Emerald, IEEExplore, ScienceDirect, Springer y Wiley han proporcionado a las cuatro universidades públicas. Los resultados muestran que las revistas de LIS se adscriben además a otras categorías y, por tanto, recogen una investigación pluridisciplinar. Los investigadores de Documentación no colaboran, sin embargo, de manera extensiva con otras disciplinas. Se confirma que los proveedores analizados distribuyen títulos que interesan a las comunidades académicas investigadas, aunque las preferencias no son homogéneas. Se constata, finalmente, la correlación entre descargas y citas lo que demuestra que ambas métricas sirven para identificar las revistas prioritarias para los académicos.
\end{abstract}

Palabras clave: big deals; Castilla y León; citas; descargas; Documentación; producción científica; revistas electrónicas; Scopus; universidades públicas; 2011-2017.

\section{Relationship between downloads and cites of academic journals in the field of Library and Information Science: the case of public universities in Castilla y León}

\begin{abstract}
The usefulness of the electronic journals from the big deals subscribed by the public universities of the Autonomous Community of Castilla y León (University of Burgos, University of León, University of Salamanca and University of Valladolid) is investigated based on the analysis of downloads and citations made by their researchers. The analysis is limited to the scientific production present in Scopus in the Library \& Information Science (LIS) category during the period 2011-2017 and to download statistics that Emerald, IEEExplore, ScienceDirect, Springer and Wiley have provided to the four public universities. The results show that LIS journals also belong to other categories and, therefore, collect multidisciplinary research. However, Information Science researchers do not collaborate extensively with other disciplines. It is confirmed that the analyzed providers distribute titles that interest the academic communities investigated, although the preferences are not homogeneous. Finally, the correlation between downloads and citations is verified, which shows that both metrics serve to identify the priority journals for academics.
\end{abstract}

Keywords: big deals; Castilla y León; citations; downloads; Information Science; scientific production; electronic journals; Scopus; public universities; 2011-2017.

Copyright: $\odot 2021$ CSIC. Este es un artículo de acceso abierto distribuido bajo los términos de la licencia de uso y distribución Creative Commons Reconocimiento 4.0 Internacional (CC BY 4.0). 


\section{INTRODUCCIÓN}

La facilidad de acceso a los recursos de información fue puesta de relieve ya hace casi una década como una prioridad para los investigadores (Connaway y otros, 2011). En este sentido, Tenopir y otros (2011) subrayan que cuando los investigadores ponen en una balanza los factores que les conducen a decidir qué artículos leer, el segundo factor, por detrás del tema del artículo, es la facilidad de acceso online. En el caso de los jóvenes investigadores, según una encuesta reciente ( $\mathrm{Ni}$ cholas y otros, 2020), la facilidad para acceder a una publicación es el principal factor que les conduce a su lectura.

La contratación de big deals por parte de las bibliotecas universitarias durante las últimas dos décadas ha resultado, por tanto, conveniente para los investigadores. La suscripción de paquetes de revistas a los principales editores ha desempeñado un papel clave en el acceso a la información electrónica por parte de la comunidad académica, lo que ha permitido a los investigadores elegir contenidos entre un considerable abanico de revistas, siendo ampliamente conocido que las necesidades de los académicos son muy diversas, incluso en la misma área de conocimiento. La abundancia de contenidos en esos paquetes ha permitido a la biblioteca transitar desde el modelo de "sé lo que necesitan mis usuarios" al de "vamos a ver qué necesitan mis usuarios", como apuntó Sanville (2001).

Sin embargo, el modelo big deal, generalizado en las bibliotecas, ha devenido en colecciones casi clónicas, sin relación con las necesidades específicas de las distintas universidades y ha convertido en marginal el proceso tradicional de desarrollo de la colección. En este escenario, la necesidad de identificar la utilidad y el valor de las colecciones suscritas radica en la rendición de cuentas que las bibliotecas deben a sus instituciones. Cuando el Plan S (https://www.coalition-s.org) dirige la comunicación científica hacia el acceso abierto, las bibliotecas han de justificar con datos cuantitativos el valor de las colecciones contratadas en las que invierten sus presupuestos, existiendo para ello diferentes métodos que van desde el análisis de las descargas hasta el análisis de citas en la producción científica, siendo también habitual que se combinen con indicadores de costes.

Un análisis reciente de las descargas realizadas por los investigadores de las cuatro universidades públicas de la Comunidad Autónoma de Castilla y León (universidades de Burgos, León, Salamanca y Valladolid) de las revistas de los principales big deals suscritos -Elsevier, Emerald, Springer y Wiley- (Fernández-Ramos y otros, 2019) ha detec- tado que las descargas de las revistas contratadas se han incrementado en los últimos años (20122018), siendo el suministrador de Elsevier, ScienceDirect, el más utilizado con mucha diferencia con respecto al resto. Pensamos que este resultado guarda relación con el acceso conveniente -transparente y directo- que los investigadores tienen a los recursos suscritos, tan sencillo que muchos no son conscientes de que ese acceso que su universidad les proporciona tiene un coste y elevado. Esta situación se produce a pesar de que el número de profesores y estudiantes ha declinado en el intervalo estudiado y pese a la proliferación de revistas en acceso abierto, de repositorios y de plataformas como Sci-Hub, que están generando nuevas formas de acceso a la información científica con un peso creciente en la comunidad académica (Himmelstein y otros, 2018; González-Solar y Fernández-Marcial, 2019; Nicholas y otros, 2019).

Aunque a priori la demanda de información es elástica, debido a la variedad de preferencias de los investigadores, la búsqueda de información se concentra en un número limitado de títulos reputados y ligados a la obtención de estabilidad y/o promoción en la academia (Shu y otros, 2018). Por tanto, gran parte de los títulos que distribuyen los proveedores recaban poco o ningún uso. Acordes con esta situación general, las universidades públicas de Castilla y León utilizan un limitado número de títulos de los paquetes suscritos y realizan de la mayoría de ellos un reducido número de descargas (Fernández-Ramos y otros, 2019). Se ha considerado que el incremento de descargas constatado, probablemente lleve aparejado un aumento de la lectura y la cita de artículos de estas revistas, lo que ha suscitado el interés por verificar si este repunte en el uso de las revistas electrónicas suscritas va acompañado de un incremento en la utilización de las mismas en las publicaciones de sus investigadores a través de las citas que realizan. En este trabajo, por tanto, se pretende indagar en el uso que se hace de las revistas contratadas a partir del análisis de su utilización en la producción científica de los investigadores, compararlo con los datos de descargas y así poder evaluar la posible relación entre descargas y citación.

\section{REVISIÓN DE LA LITERATURA}

Son numerosos los estudios referidos a bibliotecas universitarias que han analizado la adecuación de sus colecciones a las necesidades de los usuarios, principalmente a través de las estadísticas de descargas o de las referencias citadas, tal y como recogen Kurtz y Bollen (2010). Los estudios de las estadísticas de descargas tienen como referente los trabajos de David Nicholas y su grupo de in- 
vestigación CIBER (CIBER, 2009), existiendo también en España grupos de investigación que han mostrado un interés sostenido en este método de análisis para evaluar la utilidad de las colecciones electrónicas, así como para analizar su rentabilidad y repercusión en la productividad científica (Urbano y otros, 2004; Rodríguez-Bravo y otros, 2008, 2012a, 2012b; Rodríguez-Bravo y Alvite-Díez, 2006a, 2006b, 2011, 2013; Fernández-Ramos y otros, 2019).

El análisis de citas para demostrar el valor de las colecciones ha sido ampliamente empleado desde los años 70, como refleja la literatura especializada (Chambers y Healey, 1973). Destaca el trabajo de Wilson y Tenopir (2008), quienes utilizan una muestra de revistas y sus referencias combinando los datos obtenidos con los resultados de una encuesta sobre tendencias en la lectura para evaluar las colecciones de una biblioteca universitaria, así como el trabajo de Salisbury y Smith (2010), que utilizan la Web of Knowledge para identificar las revistas donde sus profesores publican y las revistas que citan en ellas. Con frecuencia estos estudios se han focalizado en las publicaciones de los investigadores de disciplinas particulares, pero también encontramos análisis de las referencias de varias disciplinas o de varias instituciones, como ponen de relieve Belter y Kaske (2016) en su estudio centrado en las Ciencias Atmosféricas y Oceánicas. Un ejemplo de análisis multidisciplinar es el de Currie y Monroe-Gulick (2013) realizado en la universidad de Kansas. Cabe mencionar igualmente la revisión de esta metodología realizada por Hoffman y Doucette (2012), quienes, tras analizar 34 estudios de análisis de referencias publicados entre los años 2005 y 2010 para evaluar su transparencia y el rigor de sus metodologías, constatan que la mayoría no proporciona suficientes detalles de sus métodos y resultados como para permitir la comparación con otros trabajos o su replicabilidad.

A pesar del interés de cada una de estas metodologías, también presentan limitaciones bien conocidas. Bucknell (2012) señala diferentes circunstancias que no aconsejan la utilización de las estadísticas de descargas como única medida para evaluar las revistas y/o colecciones contratadas. Apunta que son varios los factores que dificultan la asignación de valor a las revistas: el diseño de las plataformas, la variedad de títulos incluidos en los paquetes, la cantidad de contenido de las revistas, los cambios asociados a éstas -cambios de título, plataforma, editor-, y la dificultad de asignar precios a los ítems. Indica, acertadamente, que las estadísticas necesitan una revisión minuciosa de anomalías peculiares antes de darlas por válidas. Asimismo, como Nicholas (2008) ha puesto de re- lieve, las descargas no siempre implican lecturas ni un aprovechamiento real del artículo. Medeiros (2007) se manifiesta en contra de basar la evaluación de colecciones únicamente en las estadísticas de uso, porque éstas muestran más la utilidad de los recursos que se evalúan que su valor, es decir, con qué propósito un recurso es usado.

Otra dificultad para valorar las colecciones reside en que el uso de los artículos varía de finalidad y de intensidad según las disciplinas y, por tanto, sin conocer el entorno de los datos no es posible atribuir un valor aquilatado. En este sentido, Luther (2002) señala que las estadísticas de uso sin contexto tienen un valor relativo: "es peligroso asumir que un título popular porque es utilizado por los estudiantes es más valioso que una revista especializada utilizada por pocos investigadores de una disciplina específica".

Respecto a los análisis de citas, son conocidas sus limitaciones, a saber, la extensión del contenido cubierto por las bases de datos de citas, la imposibilidad de conocer si los investigadores están citando un artículo haciendo de él una valoración positiva o negativa, y la inconsistencia de las prácticas de citación de los investigadores de diferentes disciplinas (Martin y otros, 2016). Por otra parte, este tipo de análisis es muy complejo, exige mucho tiempo y hay mucha heterogeneidad de procedimientos para llevarlo a cabo, que frecuentemente redundan en dificultades para que puedan ser replicados (White, 2019).

Martin y otros (2016) defienden que mientras que existen muchos ejemplos en la literatura que confían en los datos de uso o en los análisis de citas para tomar decisiones sobre las colecciones de revistas, son escasos los estudios que reúnen conjuntos de datos que combinan ambos métodos evaluativos. Aludiremos, por tanto, a algunos antecedentes en esta línea de trabajo mencionando estudios que han examinado las estadísticas de uso de una colección de revistas y han efectuado un análisis de citas de la actividad investigadora de la misma institución para obtener una visión más amplia y precisa de cómo se está utilizando una colección.

Antes de la existencia de las estadísticas de uso normalizadas (COUNTER), Tsay (1998) comparó el uso de las revistas de una biblioteca médica con las citas de los investigadores de la institución durante el mismo periodo, afirmando que existía una relación significativa estadísticamente entre la frecuencia de uso y el número de citas a revistas de ciencias médicas. Otro estudio temprano que sugiere que existe una correlación entre las citas y otras medidas de uso de revistas es el de Blecic 
(1999) sobre el ámbito de ciencias de la salud en la Universidad de Illinois (Chicago). Igualmente, tras revisar las estadísticas COUNTER del Instituto de Tecnología de California, McDonald (2007) afirma que el uso de las revistas en formato online es una variable significativa para predecir patrones de citas.

Martin y otros (2016), refiriéndose a las métricas de coste-por-descarga y coste-por referencia para evaluar colecciones, subraya que ambas no se pueden comparar. Descargar un artículo es diferente de citar un artículo, requiere un esfuerzo menor. Así, es probable que el número de descargas de un título muy utilizado sea muy superior al número de citas de un artículo muy citado. Además, aunque estos datos van cambiando con el tiempo, la evolución no será necesariamente paralela, dado que las referencias citadas probablemente se retrasen con respecto a la descarga del artículo debido al tiempo que media entre la consulta de un contenido y la publicación del artículo que lo cita.

Ivanov y otros (2020) afirman que el valor de las revistas no se logra capturar solo por métodos cuantitativos, sino que requiere la combinación con otros cualitativos como consultas a los stakeholders por medio de encuestas o focus groups, por ejemplo. Estos mismos autores apoyan, no obstante, la combinación de métodos cuantitativos como indicadores del valor intelectual de una revista (identificando la frecuencia en que sus artículos son citados) y de la utilidad de una publicación (identificando la frecuencia con que los artículos de una revista son consultados y descargados).

\section{OBJETIVOS}

El objetivo general de este trabajo es indagar en la utilidad de las revistas electrónicas de los principales proveedores contratados por las universidades públicas de la Comunidad Autónoma de Castilla y León a partir del análisis de su citación en la producción científica de sus académicos, compararlo con los datos de descargas y así poder constatar la posible relación entre descargas y citación. Para ello, se ha llevado a cabo una primera aproximación a partir de un estudio de caso, en el que se ha restringido la investigación a la producción científica de las cuatro universidades públicas de Castilla y León recogida en las revistas incluidas en la categoría Library and Information Sciences (LIS) de Scopus en el periodo 2011-2017.

Para concretar los aspectos relevantes de la producción científica analizada, los patrones de citación y la relación entre citas y descargas, este objetivo general se articuló en los siguientes objetivos específicos:
- Analizar esta producción científica desde el punto de vista de la autoría y conocer quiénes son los autores de estos trabajos, de qué ámbito temático provienen y cómo colaboran entre ellos.

- Analizar las referencias bibliográficas utilizadas en esa producción científica y ver así qué pautas de citación se siguen, especialmente en lo relativo a las citas a artículos de revista.

- Observar si las revistas más citadas en estas universidades pertenecen a los principales proveedores contratados o si, por el contrario, los investigadores se centran en revistas ajenas a los suministradores estudiados.

- Estudiar la posible correlación entre el número de citas y el de descargas de las revistas suscritas. Es decir, averiguar si las revistas más citadas también son las más descargadas o si por el contrario no hay relación entre ambas variables.

\section{METODOLOGÍA}

La metodología utilizada en este trabajo es observacional y de tipo cuantitativo y se ha basado fundamentalmente en la búsqueda en Scopus de la producción científica objeto de análisis, en la descarga y normalización de los registros bibliográficos, en el análisis de las referencias bibliográficas utilizadas y en su contraste con los datos de las estadísticas de descargas. Las etapas seguidas se muestran a continuación.

\subsection{Obtención y normalización de registros de producción científica}

En primer lugar, se identificó la producción científica de las universidades públicas de Castilla y León incluida en la categoría Library and Information Sciences en Scopus durante el periodo comprendido entre 2011 y 2017. Se eligió esta base de datos en vez de la Web of Science debido a su mayor cobertura en términos globales y en particular en el ámbito de las Ciencias Sociales. La búsqueda se llevó a cabo en julio de 2018 para cada una de las cuatro universidades públicas de Castilla y León utilizando el nombre de la universidad en el campo afiliación y posteriormente se seleccionaron los registros que se correspondían con fuentes incluidas en la categoría LIS. El total de documentos recuperados fue de 186: 115 de la Universidad de Salamanca (USAL), 37 de la de Valladolid (UVA), 18 de la de León (ULE) y 16 de la de Burgos (UBU).

Hay que tener en cuenta que las universidades de Salamanca y Valladolid son universidades grandes con una considerable oferta de titulaciones y 
una comunidad académica acorde, mientras que las universidades de León y Burgos son instituciones de tamaño inferior. El número de registros está en consonancia también con la existencia de personal del área de conocimiento de Biblioteconomía y Documentación en las Universidades de León y Salamanca, puesto que únicamente estas universidades imparten titulaciones directamente vinculadas con dicha disciplina. Se trata del Grado en Información y Documentación, que se oferta en ambas, y del Máster Universitario en Sistemas de Información Digital, que se imparte en la Universidad de Salamanca.

Los registros bibliográficos fueron descargados de Scopus en formato csv e importados a un fichero de Excel. Antes de proceder a su análisis, se llevó a cabo una revisión y depuración de los mismos con el fin de asegurarnos que los documentos correspondían a autores de las universidades incluidas en el estudio. Esta revisión manual fue muy esclarecedora, ya que permitió desechar 13 registros. El total de registros incluidos fue de 167, pues 6 de los 173 registros restantes fueron escritos en colaboración entre autores de dos universidades. En concreto, el número de los registros correspondientes a cada universidad, incluyendo esos 6 registros con autores de más de una universidad fueron: 16 de UBU, 18 de ULE, 102 de USAL y 37 de UVA.

\subsection{Análisis de autoría y colaboración}

En la base de datos conformada por esos 167 registros fue necesario llevar a cabo una normalización de los autores y de sus afiliaciones y una posterior clasificación en áreas temáticas de adscripción. Esta clasificación se hizo a partir del campo filiación de los autores, haciendo un análisis de contenido y agrupándolos en diez bloques: Biblioteconomía y Documentación (ByD); Informática (INF); Economía y Empresa (ECON); Comunicación y Periodismo (COM); Humanidades (HUM); Ingenierías (ING); Ciencias; Ciencias Sociales (CC. SOC); Ciencias de la Salud (CC. SALUD); y Otros, donde se incluyeron los que no encajaban en ninguna de las categorías anteriores.

Se obtuvieron las frecuencias y porcentajes de autoría, tanto a nivel individual como por áreas temáticas, así como la tasa de colaboración, distribución de obras por autor y número medio de autores por documento. Para poder observar mejor las relaciones entre los distintos autores y áreas temáticas, se utilizó el software Vosviewer, que permite representar gráficamente esas relaciones de coautoría haciendo uso de técnicas de análisis de redes sociales.

\subsection{Análisis de referencias bibliográficas}

Al extraer las referencias bibliográficas de estos 167 registros, se encontraron también algunas pequeñas anomalías que obligaron a una normalización manual de algunos registros. Finalmente, se extrajeron las 6.465 referencias bibliográficas contenidas en 163 registros (hubo cuatro de ellos que no contenían ninguna referencia) y fueron analizadas manualmente para identificar la tipología documental de cada una de ellas. El análisis se centró en las referencias a artículos de revistas, que fueron un total de 3.394. De estas referencias bibliográficas se extrajeron los títulos de las revistas y se normalizaron. Se consultó si las revistas citadas estaban indizadas en Scopus y las que sí lo estaban fueron clasificadas según las categorías de dicha base de datos. Con estos datos se obtuvieron las distribuciones de revistas citadas, tanto en términos absolutos como según su categoría temática.

\subsection{Acopio de estadísticas de descargas}

Los datos de descargas de Emerald, IEEExplore, ScienceDirect, Springer y Wiley del periodo 2011-2017 fueron proporcionados en 2018 por las bibliotecas de las universidades públicas de Castilla y León. Estos proveedores fueron contratados por el consorcio BUCLE ya en los comienzos de la primera década del siglo y se han mantenido suscritos sin interrupción, salvo en el caso de IEEXplore, que no todas las universidades han tenido contratado ininterrumpidamente en estas dos décadas. ScienceDirect, producto de la editorial Elsevier, al igual que los big deals de las editoriales Springer y Wiley, son paquetes de contenidos electrónicos pluridisciplinares y suministran un número considerable de revistas electrónicas que superan las 2000. Contrariamente, Emerald es un distribuidor especializado en ciencias sociales y de un tamaño mucho más reducido, al igual que IEEExplore, especializado en ingenierías.

Los datos se han obtenido a partir de informes COUNTER Journal Report 1 (JR1- Number of Successful Full-Text Article Requests by Month and Journal) facilitados por los proveedores. Se trata de ficheros Excel anuales con los datos detallados de descargas mensuales de artículos a texto completo desglosados por títulos de revistas.

\subsection{Análisis de la relación entre citas y des- cargas}

Una vez obtenidos los datos de citación de revistas, y contando con los datos de suscripción y descargas de los big deals contratados a los cin- 
co proveedores incluidos en el estudio, se procedió a averiguar si existía relación entre los datos de citación, suscripción y descargas. Para ello, en primer lugar, se cotejó si las revistas citadas estaban incluidas en dichos big deals. Posteriormente, y limitando a las revistas incluidas por Scopus en LIS, se compararon los datos de citación y de descargas de las revistas incluidas en los big deals sucritos. Como procedimiento para establecer la relación entre descarga y cita se partió del dato de descargas del año anterior al de publicación del documento fuente. Es decir, si un investigador de la Universidad de Salamanca publica en 2015 un documento $X$ citando una revista $Y$, se utilizaron los datos de descarga de esa revista Y en Salamanca en el año 2014. Estos datos se analizaron de forma descriptiva y se halló la correlación lineal de Pearson existente entre citas y descargas para ver el grado de relación entre las dos variables.

Es evidente que la descarga de un artículo citado puede haberse realizado el año anterior, dos años antes o más atrás e incluso el mismo año que el artículo publicado, gracias al sistema de Early View que algunas revistas han implementado en los últimos años. Es más, la fecha de descarga puede ser incierta dado que los investigadores pueden reutilizar en sus publicaciones citas que ya han hecho en otras anteriores. Con todo, consideramos que en estos años se han acortado los plazos de publicación y que la referencia de las descargas de un año anterior pudiera ser el dato más certero.

\section{RESULTADOS}

\subsection{Producción científica}

Los resultados que se muestran a continuación presentan los datos descriptivos del análisis de los 167 documentos distintos incluidos en el estudio. Estos documentos han sido publicados en 44 fuentes distintas, destacándose una gran concentración de esta producción en unas pocas: más del $50 \%$ de los documentos se corresponden con únicamente 5 fuentes: 4 revistas (El Profesional de la Información, Scire, Investigación Bibliotecológica y Revista Española de Documentación Científica) y una serie monográfica (Intelligent Systems Reference Library). Asimismo, se ha observado un predominio de las revistas españolas y latinoamericanas, contabilizándose un total de 6 revistas entre las 10 más escogidas para publicar. En la Tabla I se ofrece la distribución de las fuentes que publicaron al menos dos de estos 167 documentos.
Tabla I. Fuentes de la producción científica analizada

\begin{tabular}{|l|c|}
\hline FUENTE & N.0 DOCS. \\
\hline El Profesional de la Información & 36 \\
\hline Intelligent Systems Reference Library & 13 \\
\hline Scire & 12 \\
\hline Investigación Bibliotecológica & 9 \\
\hline $\begin{array}{l}\text { Revista Española de Documentación } \\
\text { Científica }\end{array}$ & 9 \\
\hline BiD & 6 \\
\hline $\begin{array}{l}\text { IEEE Transactions on Information } \\
\text { Theory }\end{array}$ & 6 \\
\hline Terminology & 6 \\
\hline Cuadernos.info & 5 \\
\hline Journal of Information Science & 5 \\
\hline Online Information Review & 5 \\
\hline Anales de Documentación & 4 \\
\hline Electronic Library & 4 \\
\hline Ibersid & 4 \\
\hline $\begin{array}{l}\text { Information Resources Management } \\
\text { Journal }\end{array}$ & 3 \\
\hline $\begin{array}{l}\text { International Journal of Geographical } \\
\text { Information Science }\end{array}$ & 3 \\
\hline Program & 2 \\
\hline $\begin{array}{l}\text { Revista General de Información y } \\
\text { Documentación }\end{array}$ & 2 \\
\hline Scientometrics & 3 \\
\hline Knowledge Organization & 2 \\
\hline Library Trends & 3 \\
\hline Social Science Computer Review & 3 \\
\hline
\end{tabular}

Con respecto a la autoría de estos 167 documentos, se han identificado 282 autores únicos, provenientes de diferentes disciplinas y áreas temáticas. En la Figura 1 puede observarse la adscripción de estos autores, lo que refleja claramente el carácter multidisciplinar de las publicaciones incluidas en la categoría LIS. Aunque el sector de Documentación sea el más numeroso, con el $27 \%$ de los autores, también es destacable la presencia de autores de otras áreas como Comunicación, Informática, Economía y Empresa y Humanidades, todas ellas con al menos un $10 \%$ de los autores. En el caso de las Humanidades conviene precisar que mayoritariamente se trata de autores de Traducción e Interpretación y de Filologías, en la rama de Lengua. Curiosamente, una disciplina que ha tenido tradicionalmente vinculación con la Documentación, como es la Historia, no ha tenido presencia. 
Figura 1: Áreas temáticas de adscripción de los autores

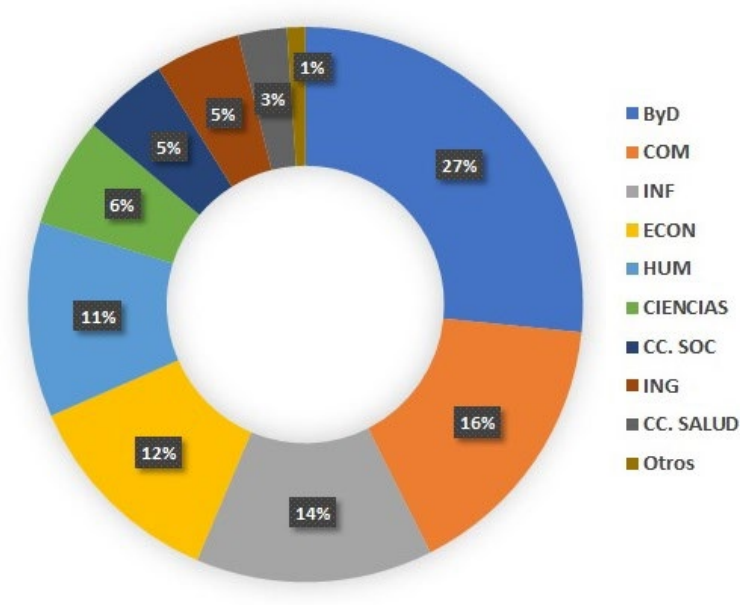

Se ha confirmado una previsible concentración de la producción científica en un número limitado de autores y un elevado número de autores con muy poca producción. Así, el 78\% de los 282 autores identificados únicamente ha firmado un documento, el $11 \%$ tiene la autoría de dos obras y son unos pocos autores los que han firmado más de tres. Con respecto a la coautoría, cabe destacar que el número medio de autores por publicación es de 2,82, siendo la mediana y la moda de 3 autores. En la Figura 2, que muestra la distribución del número de autores por artículo, se aprecia un elevado grado de coautoría ya que únicamente el $13,8 \%$ de los artículos son de autoría única, así como que la gran mayoría de los artículos han sido firmados por 2 o 3 autores, mientras que solo el $20 \%$ contaba con un número superior de firmantes. Como aspecto destacable se identificaron 36 autorías del ámbito profesional bibliotecario. Aunque en cuatro casos estos firmaron artículos en solitario, la colaboración más común entre profesionales y académicos fue aquella en la que la procedencia de estos últimos era el área de conocimiento de Biblioteconomía y Documentación (23 artículos, esto es, un $13,77 \%$ del total).

Si se analiza con más detalle esta colaboración, a partir del análisis de redes, se pueden detectar cuáles son los autores que más frecuentemente colaboran entre sí, los más prolíficos y su adscripción temática. En la Figura 3 se puede observar que hay una serie de autores que publican conjuntamente de forma habitual, destacándose por su tamaño y por el número de publicaciones tres grupos conformados básicamente por personal del ámbito de la Documentación (en color rojo), y que tienen en algunos casos cierta relación con investigadores del ámbito de la Informática (en color verde). Estos grupos de autores o redes de colaboración son el de la Universidad de León, que se establece en torno a Rodríguez-Bravo, y dos de la Universidad de Salamanca, uno liderado por Cordón-García y otro que tiene como investigador

Figura 2: Número de autores por artículo

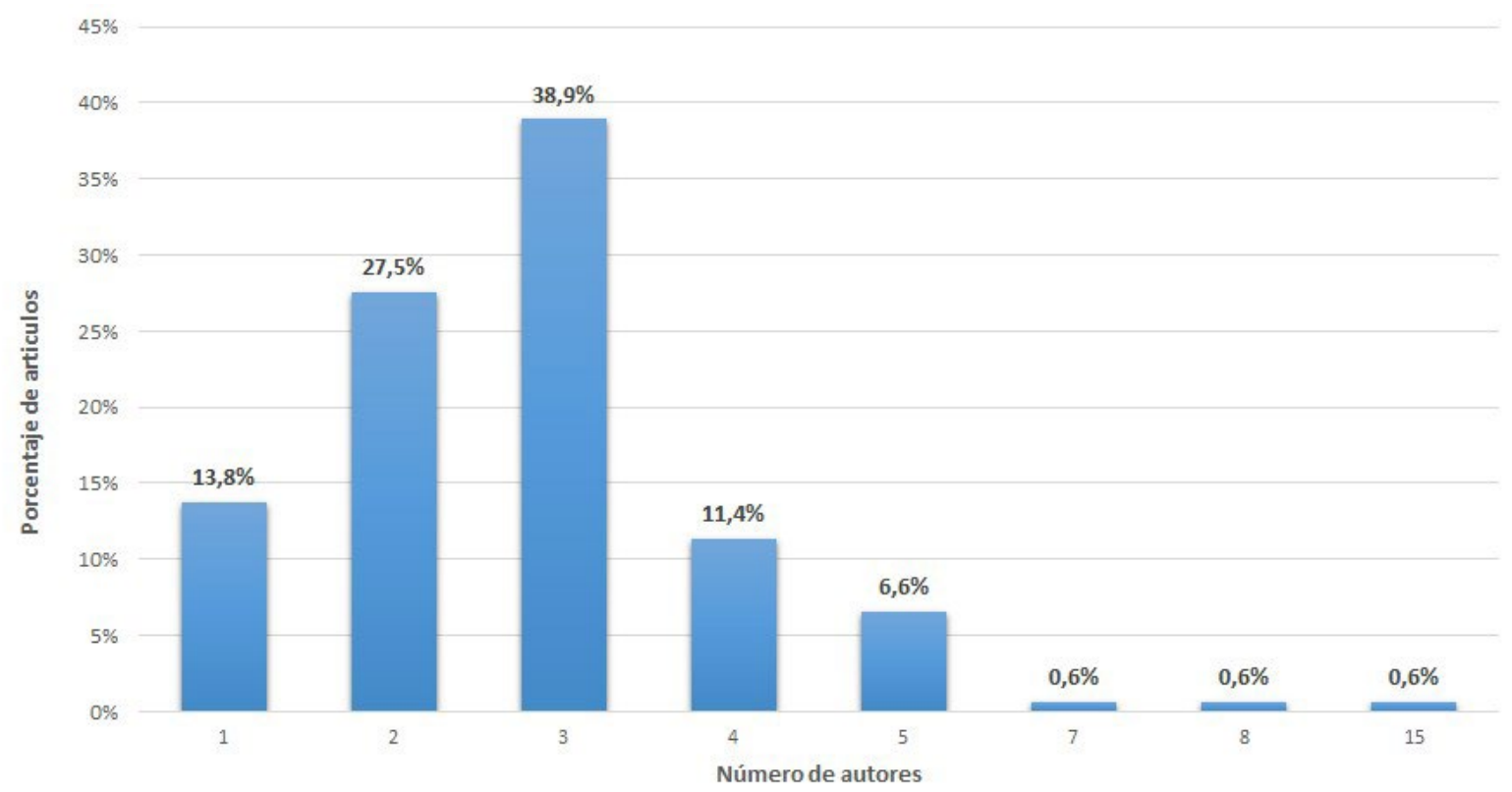


central a Ríos-Hilario. El grupo de Cordón-García ha colaborado de manera estable con otro conformado por académicos de Informática liderado por García-Figuerola.

Se aprecia también un grupo bastante numeroso y cohesionado de autores del ámbito de la Comunicación y un grupo conformado por profesores de Ingeniería. El panorama se completa con otra serie de autores de diversas disciplinas que publica menos y colabora en menor medida.

Si atendemos a los autores más prolíficos, los 16 que tienen 5 o más artículos publicados, tenemos que señalar que la mitad pertenecen al ámbito de la Documentación: 6 de ellos a la Universidad de Salamanca, todos académicos salvo un bibliotecario, y dos son investigadores de la Universidad de León. Los 8 restantes son autores que se adscriben a las Ciencias Técnicas, en concreto 7 son del ámbito de la Informática, cuatro de ellos de Salamanca y uno de Valladolid. Los dos restantes son autores de universidades andaluzas que han colaborado con un profesor de Ingeniería de la Universidad de Burgos.

\subsection{PAUTAS DE CITACIÓN}

Las referencias extraídas de la producción científica analizada suman un total de 6.465 , lo que para los artículos incluidos en el análisis -de los 167 hubo cuatro que no contaban con ninguna referencia- supone una media de casi 40 trabajos citados por documento $(39,66)$. Algo más de la mitad de estas referencias $(52,49 \%)$ son artículos de publicaciones periódicas. De estas, el 42,69\% (1.449 referencias) se corresponden con citas a revistas suscritas por las bibliotecas universitarias incluidas en este estudio, mientras que el otro $57,31 \%$ (1.945 referencias) citaban artículos de revistas no suscritas o en acceso abierto. Si tomáramos el total de referencias recogidas sin tener en cuenta si se tratan de artículo u otro tipo de documento (6.465), obtendríamos que algo menos de un cuarto del total $(22,89 \%)$ eran investigaciones publicadas en revistas de las que se tenía licencia.

En cuanto a las publicaciones, un tercio de las referencias se concentraban en las 49 revistas más citadas; mientras que para obtener la mitad de todas las referencias habría que reunir las 125 revistas con más citas. Tal y como se puede ver en la Tabla II, 66

Figura 3: Red de coautorías

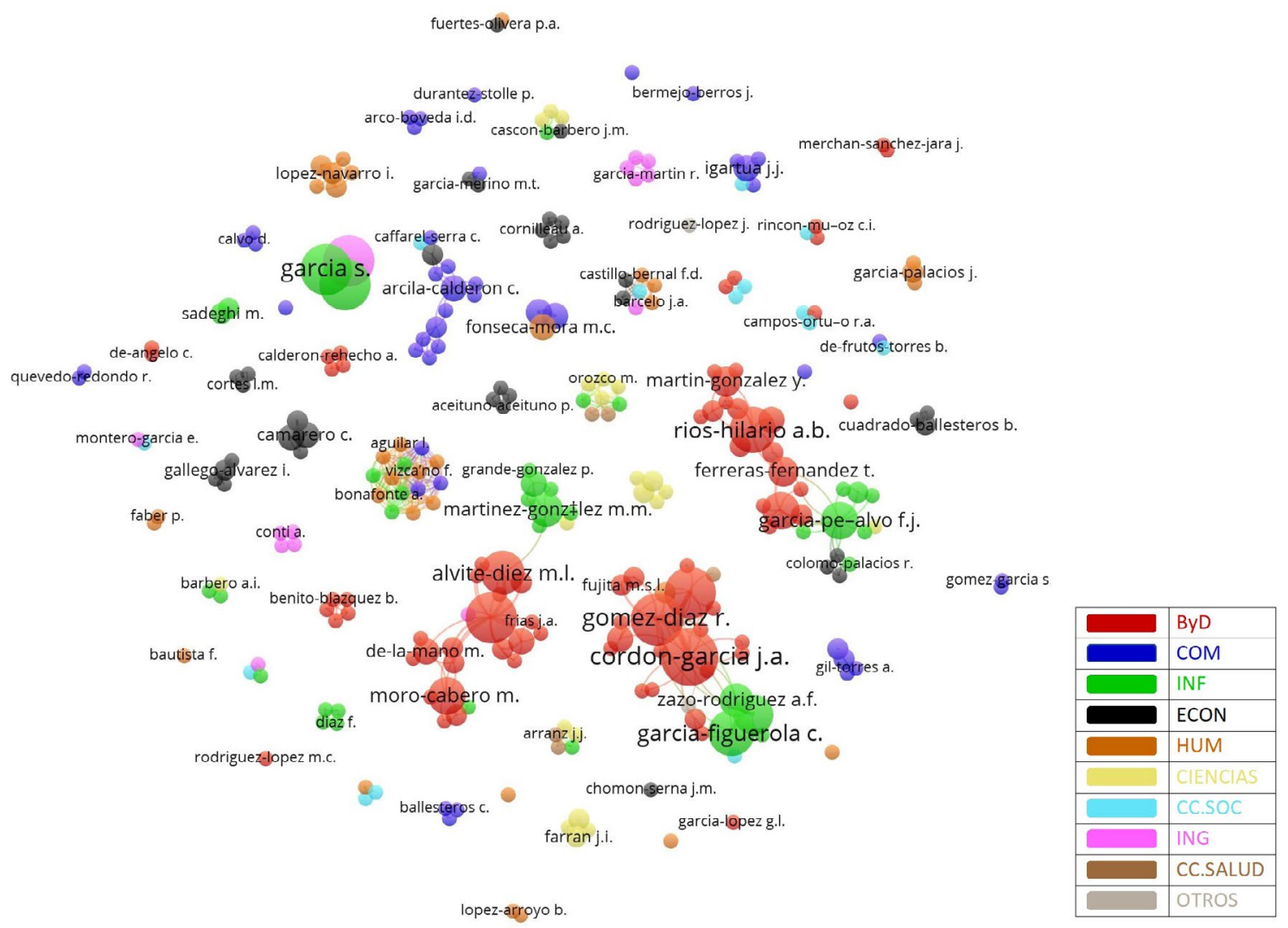


Tabla II. Revistas más citadas y proveedor

\begin{tabular}{|c|c|c|}
\hline REVISTA & CITAS & PROVEEDOR \\
\hline El Profesional de la Información & 92 & - \\
\hline JASIST + JASIS & 78 & Wiley \\
\hline Scientometrics & 62 & Springer \\
\hline $\begin{array}{l}\text { IEEE Transactions on Information } \\
\text { Theory }\end{array}$ & 49 & IEEExplore \\
\hline Pattern Recognition & 41 & Elsevier \\
\hline $\begin{array}{l}\text { Revista Española de } \\
\text { Documentación Científica }\end{array}$ & 38 & - \\
\hline $\begin{array}{l}\text { IEEE Transactions on Knowledge } \\
\text { and Data Engineering }\end{array}$ & 36 & IEEExplore \\
\hline $\begin{array}{l}\text { IEEE Transactions on Pattern } \\
\text { Analysis and Machine } \\
\text { Intelligence } \\
\end{array}$ & 34 & IEEExplore \\
\hline Machine Learning & 31 & Springer \\
\hline Online Information Review & 29 & EmeraldInsight \\
\hline $\begin{array}{l}\text { The Journal of Academic } \\
\text { Librarianship }\end{array}$ & 28 & Elsevier \\
\hline Computers in Human Behavior & 25 & Elsevier \\
\hline $\begin{array}{l}\text { Journal of Machine Learning } \\
\text { Research }\end{array}$ & 24 & - \\
\hline Journal of Business Ethics & 23 & Springer \\
\hline Journal of Documentation & 22 & EmeraldInsight \\
\hline Pattern Recognition Letters & 21 & Elsevier \\
\hline $\begin{array}{l}\text { Scire: Representación y } \\
\text { Organización del Conocimiento }\end{array}$ & 21 & - \\
\hline Journal of Information Science & 20 & - \\
\hline $\begin{array}{l}\text { Information Processing \& } \\
\text { Management }\end{array}$ & 19 & Elsevier \\
\hline Information Sciences & 19 & Elsevier \\
\hline $\begin{array}{l}\text { Journal of English for Academic } \\
\text { Purposes }\end{array}$ & 19 & Elsevier \\
\hline Anuario ThinkEPI & 18 & - \\
\hline D-Lib Magazine & 18 & - \\
\hline Library Hi Tech & 18 & EmeraldInsight \\
\hline $\begin{array}{l}\text { IEEE Transactions on Neural } \\
\text { Networks }\end{array}$ & 18 & IEEExplore \\
\hline Bioinformatics & 17 & - \\
\hline Journal of Communication & 17 & Wiley \\
\hline The Electronic Library & 17 & EmeraldInsight \\
\hline $\begin{array}{l}\text { Data Mining and Knowledge } \\
\text { Discovery }\end{array}$ & 16 & Springer \\
\hline $\begin{array}{l}\text { Revista Latina de Comunicación } \\
\text { Social }\end{array}$ & 16 & - \\
\hline Serials Review & 16 & $\begin{array}{c}\text { Elsevier (hasta } \\
\text { 2014) }\end{array}$ \\
\hline Information Research & 15 & - \\
\hline Knowledge-Based Systems & 15 & Elsevier \\
\hline
\end{tabular}

\begin{tabular}{|c|c|c|}
\hline REVISTA & CITAS & PROVEEDOR \\
\hline Aslib Proceedings & 14 & EmeraldInsight \\
\hline Anales de Documentación & 13 & - \\
\hline $\begin{array}{l}\text { Knowledge and Information } \\
\text { Systems }\end{array}$ & 13 & Springer \\
\hline New Media and Society & 13 & - \\
\hline PLoS One & 13 & - \\
\hline Artificial Intelligence & 12 & Elsevier \\
\hline Comunicar & 12 & - \\
\hline $\begin{array}{l}\text { Expert Systems With } \\
\text { Applications }\end{array}$ & 12 & Elsevier \\
\hline $\begin{array}{l}\text { Journal of Accounting and Public } \\
\text { Policy }\end{array}$ & 12 & Elsevier \\
\hline $\begin{array}{l}\text { Library \& Information Science } \\
\text { Research }\end{array}$ & 12 & Elsevier \\
\hline $\begin{array}{l}\text { Meta: Journal des traducteurs = } \\
\text { translators' journal }\end{array}$ & 12 & - \\
\hline $\begin{array}{l}\text { Program: Electronic Library and } \\
\text { Information Systems }\end{array}$ & 12 & EmeraldInsight \\
\hline $\begin{array}{l}\text { BiD: textos universitaris de } \\
\text { biblioteconomia i documentació }\end{array}$ & 11 & - \\
\hline College \& Research Libraries & 11 & - \\
\hline $\begin{array}{l}\text { Estudios sobre el Mensaje } \\
\text { Periodístico }\end{array}$ & 11 & - \\
\hline $\begin{array}{l}\text { Information, Communication and } \\
\text { Society }\end{array}$ & 11 & - \\
\hline $\begin{array}{l}\text { Information Technology and } \\
\text { Libraries }\end{array}$ & 11 & - \\
\hline $\begin{array}{l}\text { Journal of Computer-Mediated } \\
\text { Communication }\end{array}$ & 11 & Wiley \\
\hline Journal of Informetrics & 11 & Elsevier \\
\hline $\begin{array}{l}\text { Lecture Notes in Computer } \\
\text { Science }\end{array}$ & 11 & Springer \\
\hline $\begin{array}{l}\text { IEEE Transactions on Signal } \\
\text { Processing }\end{array}$ & 11 & IEEExplore \\
\hline Strategic Management Journal & 11 & Wiley \\
\hline Tábula & 11 & - \\
\hline $\begin{array}{l}\text { Telos. Cuadernos de } \\
\text { Comunicación e Innovación }\end{array}$ & 11 & - \\
\hline English for Specific Purposes & 10 & Elsevier \\
\hline Intelligent Systems, IEEE & 10 & IEEExplore \\
\hline Journal of Business Research & 10 & Elsevier \\
\hline Learned Publishing & 10 & Wiley \\
\hline Library Journal & 10 & - \\
\hline Nature & 10 & - \\
\hline New Library World & 10 & EmeraldInsight \\
\hline Research Policy & 10 & Elsevier \\
\hline Soft Computing & 10 & Springer \\
\hline
\end{tabular}


revistas han recibido 10 o más citas. De esos títulos, 42 estaban suscritos por las universidades públicas de Castilla y León dentro de los big deals analizados, lo que representa un $63,6 \%$ del total, y de ese subconjunto, solo 15 de ellos (sombreados en la tabla) aparecen incluidas en la categoría LIS de Scopus.

Entre los proveedores que dan acceso a las revistas suscritas más útiles para la producción científica de los investigadores de Castilla y León destaca ScienceDirect, de Elsevier, que distribuye 17 de las revistas más citadas, lo que supone el $40,4 \%$ del total. Sin embargo, no son mayoría las revistas de Elsevier en la categoría LIS, sino que se limitan a únicamente cinco títulos, entre los que destaca Journal of Academic Librarianship e Information Processing \& Management, ambas revistas muy reputadas en el ámbito.

Emerald y Springer, por su parte, dan cada una de ellas acceso a siete revistas de entre las más citadas. En el caso de Emerald los siete títulos se encuadran en la categoría LIS, destacando las citas a Online Information Review y a Journal of Documentation, mientras que en el caso de Springer, solo la revista Scientometrics se adscribe a esa categoría. Wiley e IEEExplore distribuyen cada uno cinco de los títulos que más citas reciben. De entre ellos solo JASIST, en el caso de Wiley e IEEE Transactions on Information Theory de IEEE se ubican en la categoría LIS.

En el conjunto de revistas aparecen también títulos en acceso abierto, como Information Research, Information Technology and Libraries o la Revista Española de Documentación Científica, y revistas suministradas por otros distribuidores o editores prestigiosos, como es el caso de Journal of Information Science y New Media and Society de SAGE o Information, communication and Society y Serials Review de Taylor \& Francis. Completan la tabla de títulos más citados revistas de prestigio, como son Nature y Bioinformatics, megarrevistas en abierto, como PloS $O N E$, y otros títulos de distintos ámbitos temáticos, entre los que predomina la Comunicación, la Informática, la Economía y las Humanidades.

Cabe mencionar, asimismo, que existe un número no desdeñable de revistas españolas entre las más citadas $(16,6 \%)$. De ellas la mayoría cubren el ámbito de Documentación, y la minoría el de Comunicación. Se trata de títulos bien considerados, con tradición, varios de ellos incluidos en Scopus. Destacan en las primeras posiciones El Profesional de la Información y la Revista Española de Documentación Científica. Estas dos revistas están acompañadas por Scire, el Anuario ThinkEPI, Revista Latina de Comunicación Social, Anales de Documentación, Comunicar, y BiD entre otras.
Con respecto al dominio temático de las revistas citadas, se analizaron las materias asignadas a aquellas revistas que estaban incluidas en Scopus, condición que cumplían el $73,13 \%$ de las 1243 revistas identificadas, y se obtuvo una distribución por categorías temáticas que aparece recogida en la Tabla III para las 15 categorías más frecuentes de las 206 identificadas. Para interpretar esta tabla hay que tener en cuenta que una revista puede aparecer clasificada en más de una categoría; de hecho, la media de categorías asignadas por revista es de 2,29, y por eso la suma de los porcentajes superaría el $100 \%$.

Tabla III. Categorías temáticas más frecuentes en las revistas citadas

\begin{tabular}{|l|c|}
\hline CATEGORÍA TEMÁTICA & \% \\
\hline Library and Information Sciences & 31,22 \\
\hline Computer Science Applications & 14,70 \\
\hline Software & 12,50 \\
\hline Artificial Intelligence & 11,37 \\
\hline Communication & 10,10 \\
\hline Information Systems & 8,96 \\
\hline Sociology and Political Science & 6,42 \\
\hline Language and Linguistics & 6,18 \\
\hline Education & 5,98 \\
\hline Linguistics and Language & 5,77 \\
\hline $\begin{array}{l}\text { Computer Networks and } \\
\text { Communications }\end{array}$ & 4,43 \\
\hline $\begin{array}{l}\text { Computer Vision and Pattern } \\
\text { Recognition }\end{array}$ & 4,02 \\
\hline Economics and Econometrics & 3,95 \\
\hline $\begin{array}{l}\text { Computational Theory and } \\
\text { Mathematics }\end{array}$ & 3,91 \\
\hline Human-Computer Interaction & 3,43 \\
\hline
\end{tabular}

Como cabría esperar, la más habitual fue LIS, que duplica el porcentaje de aparición de la siguiente categoría. A continuación, aparecen categorías vinculadas al ámbito informático (Computer Science Applications, Software y Artificial Intelligence), cuya suma llega al 38,52\%, lo que denota la importancia de la rama en la bibliografía citada, y la categoría Communication $(10,1 \%)$. Otro aspecto destacado en las revistas referenciadas es que coinciden de forma predominante con los campos temáticos a los que pertenecen los propios autores que firman los trabajos, esto es, además de las mencionadas, a materias como Economía, Sociología, Lengua y Educación.

Si nos atenemos al nivel más genérico de clasificación que proporciona Scopus, obtenemos una 
Figura 4. Áreas temáticas de las revistas citadas

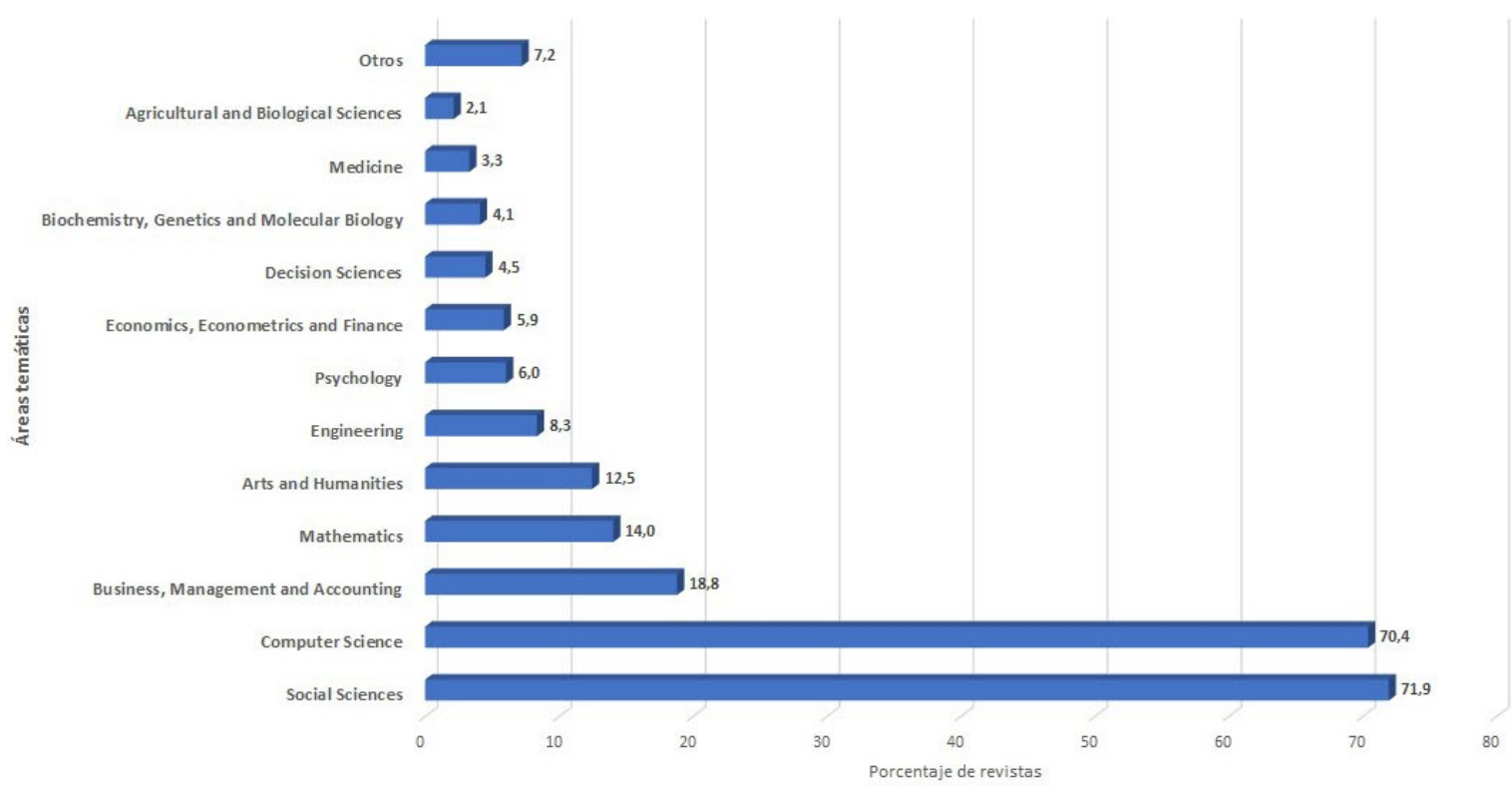

figura elocuente de las principales áreas temáticas, donde Ciencias Sociales e Informática acaparan un protagonismo compartido muy similar; el $71,9 \%$ y el $70,4 \%$, respectivamente, de las revistas que estaban encuadradas al menos en alguna de esas áreas temáticas (Figura 4). Por otro lado, se registró un amplio abanico de 24 áreas distintas al que podían pertenecer las publicaciones citadas. Bajo el epígrafe Otros se han agrupado catorce disciplinas cuya frecuencia de aparición fue menor: Environmental Science, Multidisciplinary, Physics and Astronomy Energy, Chemistry, Nursing, Earth and Planetary Sciences, Materials Science, Immunology and Microbiology, Neuroscience, Chemical Engineering, Health Professions, Pharmacology, Toxicology and Pharmaceutics y Veterinary.

\subsection{Relación entre descargas y citas}

En la Tabla IV observamos las descargas y las citas que han recibido las revistas incluidas en el análisis por parte de cada una de las cuatro universidades públicas de Castilla y León. En ella se aprecia una mayor utilización de las revistas suscritas del ámbito de la Documentación en las Universidades de Salamanca y León, que son aquellas con personal docente e investigador del área de Biblioteconomía y Documentación, siendo marginal en el caso de la Universidad de Valladolid e inexistente en la de Burgos.

Por otra parte, se aprecia, tanto a nivel conjunto como en los casos de las universidades de Salamanca y León, bastante relación entre las revistas más citadas y las más descargadas. Así, JASIST y
Scientometrics son las revistas más citadas y descargadas por parte de los investigadores de la Universidad de Salamanca, mientras que en el caso de León son JASIST, The Journal of Academic Librarianship, Information Processing \& Management y Journal of Documentation. En la Universidad de Valladolid, que no tiene personal docente e investigador propio de Biblioteconomía y Documentación, la relación entre citas y descargas no es tan evidente. Además, las revistas que más utilizan sus académicos difieren un poco con respecto a las preferencias de los investigadores de otras universidades. Así, algunas de las que más se han utilizado en esta universidad son Online Information Review, Library Hi Tech, International Journal of Information Management y Government Information Quarterly; las dos primeras de ámbito biblioteconómico y las últimas más vinculadas a la gestión.

Si analizamos la relación entre citas y descargas desde un punto de vista temporal, en la Tabla V se puede observar de nuevo la correlación entre descargas y citas en la mayor parte de los títulos y de las anualidades estudiadas. Como se puede apreciar, existe una tendencia creciente que viene a corroborar lo que ya Fernández-Ramos y otros (2019) habían puesto de manifiesto sobre el conjunto de descargas de estas universidades y para cuatro de los proveedores aquí estudiados. Los datos totales de descargas y citas de 2017 apuntan un crecimiento acorde, quizá, con una mayor producción científica de las Universidades de Castilla y León en general, y del área de Biblioteconomía y Documentación y otras áreas próximas en particular. 
Tabla IV. Descargas y citas de las revistas incluidas según universidades

\begin{tabular}{|c|c|c|c|c|c|}
\hline & UBU & ULE & USAL & UVA & TOTAL \\
\hline & $\begin{array}{c}\text { Descargas } \\
\text { Citas }\end{array}$ & $\begin{array}{c}\text { Descargas } \\
\text { Citas }\end{array}$ & $\begin{array}{c}\text { Descargas } \\
\text { Citas }\end{array}$ & $\begin{array}{c}\text { Descargas } \\
\text { Citas }\end{array}$ & $\begin{array}{c}\text { Descargas } \\
\text { Citas }\end{array}$ \\
\hline JASIST + JASIS & & $\begin{array}{c}385 \\
19\end{array}$ & $\begin{array}{c}1392 \\
54\end{array}$ & $\begin{array}{c}36 \\
5\end{array}$ & $\begin{array}{c}1813 \\
78\end{array}$ \\
\hline Scientometrics & & $\begin{array}{c}10 \\
6\end{array}$ & $\begin{array}{c}517 \\
56\end{array}$ & & $\begin{array}{c}527 \\
62\end{array}$ \\
\hline IEEE Transactions on Information Theory & $\begin{array}{l}1 \\
9\end{array}$ & & $\begin{array}{l}55 \\
12\end{array}$ & $\begin{array}{c}0 \\
28\end{array}$ & $\begin{array}{l}56 \\
49\end{array}$ \\
\hline Online Information Review & & $\begin{array}{l}5 \\
1 \\
\end{array}$ & $\begin{array}{c}459 \\
16\end{array}$ & $\begin{array}{c}131 \\
12\end{array}$ & $\begin{array}{c}595 \\
29 \\
\end{array}$ \\
\hline The Journal of Academic Librarianship & & $\begin{array}{c}466 \\
20 \\
\end{array}$ & $\begin{array}{c}141 \\
6 \\
\end{array}$ & $\begin{array}{c}29 \\
2\end{array}$ & $\begin{array}{l}636 \\
28 \\
\end{array}$ \\
\hline Journal of Documentation & & $\begin{array}{c}112 \\
10\end{array}$ & $\begin{array}{c}324 \\
12\end{array}$ & & $\begin{array}{l}436 \\
22\end{array}$ \\
\hline Information Processing \& Management & & $\begin{array}{c}137 \\
2\end{array}$ & $\begin{array}{c}245 \\
16\end{array}$ & $\begin{array}{c}33 \\
1\end{array}$ & $\begin{array}{c}415 \\
19\end{array}$ \\
\hline Library Hi Tech & & $\begin{array}{c}23 \\
6\end{array}$ & $\begin{array}{c}284 \\
9\end{array}$ & $\begin{array}{c}80 \\
3\end{array}$ & $\begin{array}{c}357 \\
18\end{array}$ \\
\hline The Electronic Library & & $\begin{array}{c}53 \\
3\end{array}$ & $\begin{array}{c}301 \\
9\end{array}$ & $\begin{array}{c}36 \\
5\end{array}$ & $\begin{array}{c}390 \\
17\end{array}$ \\
\hline Serials Review & & $\begin{array}{l}76 \\
13\end{array}$ & $\begin{array}{l}0 \\
3\end{array}$ & & $\begin{array}{l}76 \\
16\end{array}$ \\
\hline Aslib Proceedings & & $\begin{array}{c}91 \\
8\end{array}$ & $\begin{array}{c}77 \\
6\end{array}$ & & $\begin{array}{c}168 \\
14\end{array}$ \\
\hline Library \& Information Science Research & & $\begin{array}{c}106 \\
4\end{array}$ & $\begin{array}{c}272 \\
4\end{array}$ & $\begin{array}{c}37 \\
4\end{array}$ & $\begin{array}{c}415 \\
12\end{array}$ \\
\hline $\begin{array}{l}\text { Program: Electronic Library and } \\
\text { Information Systems }\end{array}$ & & $\begin{array}{c}25 \\
5\end{array}$ & $\begin{array}{c}17 \\
6 \\
\end{array}$ & $\begin{array}{c}11 \\
1\end{array}$ & $\begin{array}{l}53 \\
12 \\
\end{array}$ \\
\hline Journal of Informetrics & & & $\begin{array}{c}226 \\
11\end{array}$ & & $\begin{array}{c}226 \\
11\end{array}$ \\
\hline New Library World & & $\begin{array}{c}57 \\
6 \\
\end{array}$ & $\begin{array}{c}47 \\
3\end{array}$ & $\begin{array}{l}1 \\
1\end{array}$ & $\begin{array}{c}105 \\
10\end{array}$ \\
\hline Interlending \& Document Supply & & $\begin{array}{c}42 \\
6\end{array}$ & $\begin{array}{c}15 \\
1\end{array}$ & & $\begin{array}{c}57 \\
7\end{array}$ \\
\hline $\begin{array}{l}\text { International Journal of Information } \\
\text { Management }\end{array}$ & & & $\begin{array}{c}183 \\
4\end{array}$ & $\begin{array}{c}238 \\
3\end{array}$ & $\begin{array}{c}421 \\
7\end{array}$ \\
\hline Library Management & & & $\begin{array}{c}55 \\
7\end{array}$ & & $\begin{array}{c}55 \\
7\end{array}$ \\
\hline Archival Science & & $\begin{array}{l}0 \\
2\end{array}$ & $\begin{array}{c}49 \\
4\end{array}$ & & $\begin{array}{c}49 \\
6\end{array}$ \\
\hline Performance Measurement and Metrics & & & $\begin{array}{l}8 \\
6 \\
\end{array}$ & & $\begin{array}{l}8 \\
6\end{array}$ \\
\hline $\begin{array}{l}\text { Annual Review of Information Science and } \\
\text { Technology }\end{array}$ & & $\begin{array}{l}0 \\
1\end{array}$ & $\begin{array}{c}17 \\
4\end{array}$ & & $\begin{array}{c}17 \\
5\end{array}$ \\
\hline Library Review & & $\begin{array}{c}39 \\
1\end{array}$ & $\begin{array}{c}106 \\
3\end{array}$ & $\begin{array}{l}9 \\
1\end{array}$ & $\begin{array}{c}154 \\
5\end{array}$ \\
\hline Records Management Journal & & & $\begin{array}{c}121 \\
4\end{array}$ & & $\begin{array}{c}121 \\
4\end{array}$ \\
\hline Government Information Quarterly & & & $\begin{array}{c}53 \\
3\end{array}$ & $\begin{array}{c}137 \\
1\end{array}$ & $\begin{array}{c}190 \\
4\end{array}$ \\
\hline Investigación Bibliotecológica & $\begin{array}{l}0 \\
1\end{array}$ & $\begin{array}{c}17 \\
2\end{array}$ & & 10 & $\begin{array}{c}27 \\
4\end{array}$ \\
\hline Campus-Wide Information Systems & & & $\begin{array}{l}0 \\
1\end{array}$ & $\begin{array}{l}7 \\
2\end{array}$ & $\begin{array}{l}7 \\
3\end{array}$ \\
\hline Reference Services Review & & & $\begin{array}{c}27 \\
3\end{array}$ & & $\begin{array}{c}27 \\
3\end{array}$ \\
\hline $\begin{array}{l}\text { Library Collections, Acquisitions, and } \\
\text { Technical Services }\end{array}$ & & $\begin{array}{c}20 \\
1\end{array}$ & $\begin{array}{c}18 \\
1\end{array}$ & & $\begin{array}{c}38 \\
2\end{array}$ \\
\hline VINE & & $\begin{array}{l}6 \\
2\end{array}$ & & & $\begin{array}{l}6 \\
2\end{array}$ \\
\hline $\begin{array}{l}\text { The Bottom Line: Managing Library } \\
\text { Finances }\end{array}$ & & & $\begin{array}{c}15 \\
2\end{array}$ & & $\begin{array}{c}15 \\
2\end{array}$ \\
\hline American Archivist & & $\begin{array}{l}0 \\
2\end{array}$ & & & $\begin{array}{l}0 \\
2\end{array}$ \\
\hline Health Information \& Libraries Journal & & & $\begin{array}{c}31 \\
1\end{array}$ & & $\begin{array}{c}31 \\
1\end{array}$ \\
\hline OCLC Systems \& Services & & $\begin{array}{l}5 \\
1\end{array}$ & & & $\begin{array}{l}5 \\
1\end{array}$ \\
\hline Library Hi Tech News & & & $\begin{array}{c}33 \\
1\end{array}$ & & $\begin{array}{c}33 \\
1\end{array}$ \\
\hline Total & $\begin{array}{c}1 \\
10\end{array}$ & $\begin{array}{c}1675 \\
121\end{array}$ & $\begin{array}{c}5088 \\
268\end{array}$ & $\begin{array}{c}795 \\
70\end{array}$ & $\begin{array}{c}7559 \\
469\end{array}$ \\
\hline
\end{tabular}


Tabla V. Descargas y citas de las revistas incluidas, por años

\begin{tabular}{|c|c|c|c|c|c|c|c|c|}
\hline & 2011 & 2012 & 2013 & 2014 & 2015 & 2016 & 2017 & TOTAL \\
\hline & $\begin{array}{l}\text { Desc. } \\
\text { Citas }\end{array}$ & $\begin{array}{l}\text { Desc. } \\
\text { Citas }\end{array}$ & $\begin{array}{l}\text { Desc. } \\
\text { Citas }\end{array}$ & $\begin{array}{l}\text { Desc. } \\
\text { Citas }\end{array}$ & $\begin{array}{l}\text { Desc. } \\
\text { Citas }\end{array}$ & $\begin{array}{l}\text { Desc. } \\
\text { Citas }\end{array}$ & $\begin{array}{l}\text { Desc. } \\
\text { Citas }\end{array}$ & $\begin{array}{l}\text { Desc. } \\
\text { Citas }\end{array}$ \\
\hline JASIST + JASIS & $\begin{array}{c}689 \\
5 \\
\end{array}$ & $\begin{array}{c}200 \\
13 \\
\end{array}$ & $\begin{array}{c}108 \\
14 \\
\end{array}$ & $\begin{array}{c}161 \\
3 \\
\end{array}$ & $\begin{array}{l}63 \\
12 \\
\end{array}$ & $\begin{array}{c}40 \\
3 \\
\end{array}$ & $\begin{array}{c}552 \\
28 \\
\end{array}$ & $\begin{array}{c}1813 \\
78 \\
\end{array}$ \\
\hline Scientometrics & $\begin{array}{c}72 \\
2\end{array}$ & & $\begin{array}{l}92 \\
32\end{array}$ & & $\begin{array}{c}130 \\
7\end{array}$ & $\begin{array}{l}92 \\
10\end{array}$ & $\begin{array}{c}141 \\
11\end{array}$ & $\begin{array}{c}527 \\
62\end{array}$ \\
\hline IEEE Transactions on Information Theory & & $\begin{array}{c}0 \\
12\end{array}$ & $\begin{array}{c}55 \\
9\end{array}$ & $\begin{array}{l}0 \\
7\end{array}$ & $\begin{array}{c}1 \\
18\end{array}$ & & 0 & $\begin{array}{l}56 \\
49\end{array}$ \\
\hline Online Information Review & $\begin{array}{c}60 \\
3\end{array}$ & $\begin{array}{c}33 \\
4 \\
\end{array}$ & $\begin{array}{c}69 \\
5\end{array}$ & $\begin{array}{c}54 \\
5\end{array}$ & $\begin{array}{c}105 \\
2\end{array}$ & $\begin{array}{c}78 \\
4\end{array}$ & $\begin{array}{c}196 \\
6\end{array}$ & $\begin{array}{c}595 \\
29\end{array}$ \\
\hline The Journal of Academic Librarianship & $\begin{array}{c}40 \\
1\end{array}$ & $\begin{array}{c}217 \\
9 \\
\end{array}$ & & $\begin{array}{c}80 \\
3\end{array}$ & $\begin{array}{c}64 \\
8 \\
\end{array}$ & $\begin{array}{c}54 \\
3\end{array}$ & $\begin{array}{c}181 \\
4\end{array}$ & $\begin{array}{c}636 \\
28 \\
\end{array}$ \\
\hline Journal of Documentation & $\begin{array}{c}31 \\
4\end{array}$ & $\begin{array}{c}22 \\
3\end{array}$ & $\begin{array}{c}69 \\
6\end{array}$ & $\begin{array}{c}93 \\
1\end{array}$ & $\begin{array}{c}70 \\
3\end{array}$ & $\begin{array}{c}86 \\
2\end{array}$ & $\begin{array}{c}65 \\
3 \\
\end{array}$ & $\begin{array}{c}436 \\
22\end{array}$ \\
\hline Information Processing \& Management & $\begin{array}{c}33 \\
1\end{array}$ & $\begin{array}{c}74 \\
1 \\
\end{array}$ & $\begin{array}{c}74 \\
6 \\
\end{array}$ & $\begin{array}{c}67 \\
1\end{array}$ & $\begin{array}{c}103 \\
3\end{array}$ & $\begin{array}{c}24 \\
4 \\
\end{array}$ & $\begin{array}{c}40 \\
3\end{array}$ & $\begin{array}{c}415 \\
19 \\
\end{array}$ \\
\hline Library Hi Tech & & $\begin{array}{c}136 \\
9\end{array}$ & & $\begin{array}{c}90 \\
4\end{array}$ & & $\begin{array}{c}39 \\
1\end{array}$ & $\begin{array}{c}122 \\
4\end{array}$ & $\begin{array}{c}357 \\
18\end{array}$ \\
\hline The Electronic Library & $\begin{array}{c}13 \\
1\end{array}$ & $\begin{array}{c}39 \\
2\end{array}$ & $\begin{array}{c}78 \\
5\end{array}$ & $\begin{array}{c}62 \\
5\end{array}$ & & $\begin{array}{c}94 \\
2\end{array}$ & $\begin{array}{c}104 \\
2\end{array}$ & $\begin{array}{c}390 \\
17\end{array}$ \\
\hline Serials Review & $\begin{array}{c}25 \\
2\end{array}$ & $\begin{array}{c}48 \\
4\end{array}$ & & & $\begin{array}{l}0 \\
4\end{array}$ & $\begin{array}{l}0 \\
3\end{array}$ & $\begin{array}{l}3 \\
3\end{array}$ & $\begin{array}{l}76 \\
16\end{array}$ \\
\hline Aslib Proceedings & $\begin{array}{c}11 \\
2\end{array}$ & $\begin{array}{c}12 \\
3 \\
\end{array}$ & $\begin{array}{c}33 \\
2 \\
\end{array}$ & $\begin{array}{c}23 \\
1\end{array}$ & $\begin{array}{c}89 \\
3 \\
\end{array}$ & $\begin{array}{l}0 \\
1 \\
\end{array}$ & $\begin{array}{l}0 \\
2 \\
\end{array}$ & $\begin{array}{c}168 \\
14 \\
\end{array}$ \\
\hline Library \& Information Science Research & $\begin{array}{c}18 \\
1\end{array}$ & $\begin{array}{c}10 \\
1\end{array}$ & 150 & & $\begin{array}{c}32 \\
2\end{array}$ & & 205 & $\begin{array}{c}415 \\
12\end{array}$ \\
\hline $\begin{array}{l}\text { Program: Electronic Library and } \\
\text { Information Systems }\end{array}$ & $\begin{array}{l}4 \\
1 \\
\end{array}$ & $\begin{array}{c}30 \\
4\end{array}$ & & $\begin{array}{c}17 \\
4\end{array}$ & $\begin{array}{l}2 \\
2 \\
\end{array}$ & $\begin{array}{l}0 \\
1\end{array}$ & & $\begin{array}{l}53 \\
12 \\
\end{array}$ \\
\hline Journal of Informetrics & & & & & & $\begin{array}{c}67 \\
9\end{array}$ & $\begin{array}{c}159 \\
2\end{array}$ & 226 \\
\hline New Library World & $\begin{array}{c}31 \\
3\end{array}$ & $\begin{array}{l}8 \\
1\end{array}$ & $\begin{array}{l}7 \\
2\end{array}$ & & $\begin{array}{c}59 \\
2\end{array}$ & & $\begin{array}{l}0 \\
2\end{array}$ & $\begin{array}{c}105 \\
10\end{array}$ \\
\hline Interlending \& Document Supply & $\begin{array}{c}24 \\
3\end{array}$ & $\begin{array}{l}1 \\
4 \\
2\end{array}$ & & & $\begin{array}{c}2 \\
14 \\
1\end{array}$ & $\begin{array}{c}15 \\
1\end{array}$ & & $\begin{array}{c}57 \\
7\end{array}$ \\
\hline $\begin{array}{l}\text { International Journal of Information } \\
\text { Management }\end{array}$ & $\begin{array}{c}58 \\
2\end{array}$ & & $\begin{array}{c}72 \\
1\end{array}$ & & $\begin{array}{c}71 \\
1\end{array}$ & & $\begin{array}{c}220 \\
3\end{array}$ & $\begin{array}{c}421 \\
7\end{array}$ \\
\hline Library Management & & & $\begin{array}{c}10 \\
3\end{array}$ & $\begin{array}{c}23 \\
2\end{array}$ & & $\begin{array}{c}22 \\
2\end{array}$ & & $\begin{array}{c}55 \\
7\end{array}$ \\
\hline Archival Science & $\begin{array}{c}49 \\
4\end{array}$ & & & & & $\begin{array}{l}0 \\
2\end{array}$ & & $\begin{array}{c}49 \\
6\end{array}$ \\
\hline Performance Measurement and Metrics & & & & $\begin{array}{l}4 \\
1\end{array}$ & & $\begin{array}{l}4 \\
5\end{array}$ & & $\begin{array}{l}8 \\
6\end{array}$ \\
\hline $\begin{array}{l}\text { Annual Review of Information Science } \\
\text { and Technology }\end{array}$ & $\begin{array}{l}0 \\
1\end{array}$ & & & & & $\begin{array}{l}8 \\
1\end{array}$ & $\begin{array}{l}9 \\
3\end{array}$ & $\begin{array}{c}17 \\
5\end{array}$ \\
\hline Library Review & $\begin{array}{c}48 \\
1\end{array}$ & $\begin{array}{c}64 \\
2 \\
\end{array}$ & $\begin{array}{l}9 \\
1 \\
\end{array}$ & & $\begin{array}{c}33 \\
1\end{array}$ & & & $\begin{array}{c}154 \\
5\end{array}$ \\
\hline Records Management Journal & $\begin{array}{c}121 \\
4\end{array}$ & & & & & & & $\begin{array}{c}121 \\
4\end{array}$ \\
\hline Government Information Quarterly & & & & $\begin{array}{c}53 \\
3\end{array}$ & & & $\begin{array}{c}137 \\
1\end{array}$ & $\begin{array}{c}190 \\
4\end{array}$ \\
\hline Investigación Bibliotecológica: & & $\begin{array}{l}0 \\
1\end{array}$ & & & & & $\begin{array}{c}27 \\
3\end{array}$ & $\begin{array}{c}27 \\
4\end{array}$ \\
\hline Campus-Wide Information Systems & & $\begin{array}{l}7 \\
2\end{array}$ & & & & & $\begin{array}{l}0 \\
1\end{array}$ & $\begin{array}{l}7 \\
3\end{array}$ \\
\hline Reference Services Review & & & & & & & $\begin{array}{c}27 \\
3\end{array}$ & $\begin{array}{c}27 \\
3\end{array}$ \\
\hline $\begin{array}{l}\text { Library Collections, Acquisitions, and } \\
\text { Technical Services }\end{array}$ & $\begin{array}{c}20 \\
1\end{array}$ & & & $\begin{array}{c}18 \\
1\end{array}$ & & & & $\begin{array}{c}38 \\
2\end{array}$ \\
\hline (2) & & $\begin{array}{l}1 \\
1\end{array}$ & & & $\begin{array}{l}5 \\
1\end{array}$ & & & $\begin{array}{l}2 \\
2 \\
2\end{array}$ \\
\hline $\begin{array}{l}\text { The Bottom Line: Managing Library } \\
\text { Finances }\end{array}$ & & & & & $\begin{array}{c}15 \\
2\end{array}$ & & & $\begin{array}{c}15 \\
2\end{array}$ \\
\hline American Archivist & & & & & & $\begin{array}{l}0 \\
2\end{array}$ & & $\begin{array}{l}0 \\
2\end{array}$ \\
\hline Health Information \& Libraries Journal & & & & $\begin{array}{c}31 \\
1\end{array}$ & & & & $\begin{array}{c}31 \\
1\end{array}$ \\
\hline OCLC Systems \& Services & & & & & $\begin{array}{l}5 \\
1\end{array}$ & & & $\begin{array}{l}5 \\
1\end{array}$ \\
\hline Library Hi Tech News & & & & & & & $\begin{array}{c}33 \\
1\end{array}$ & $\begin{array}{c}33 \\
1\end{array}$ \\
\hline Total & $\begin{array}{c}1347 \\
42\end{array}$ & $\begin{array}{l}905 \\
74\end{array}$ & $\begin{array}{c}826 \\
89\end{array}$ & $\begin{array}{c}776 \\
42\end{array}$ & $\begin{array}{c}861 \\
73\end{array}$ & $\begin{array}{c}623 \\
56\end{array}$ & $\begin{array}{c}2221 \\
93\end{array}$ & $\begin{array}{c}7559 \\
469\end{array}$ \\
\hline
\end{tabular}


Figura 5: Relación entre citas y descargas

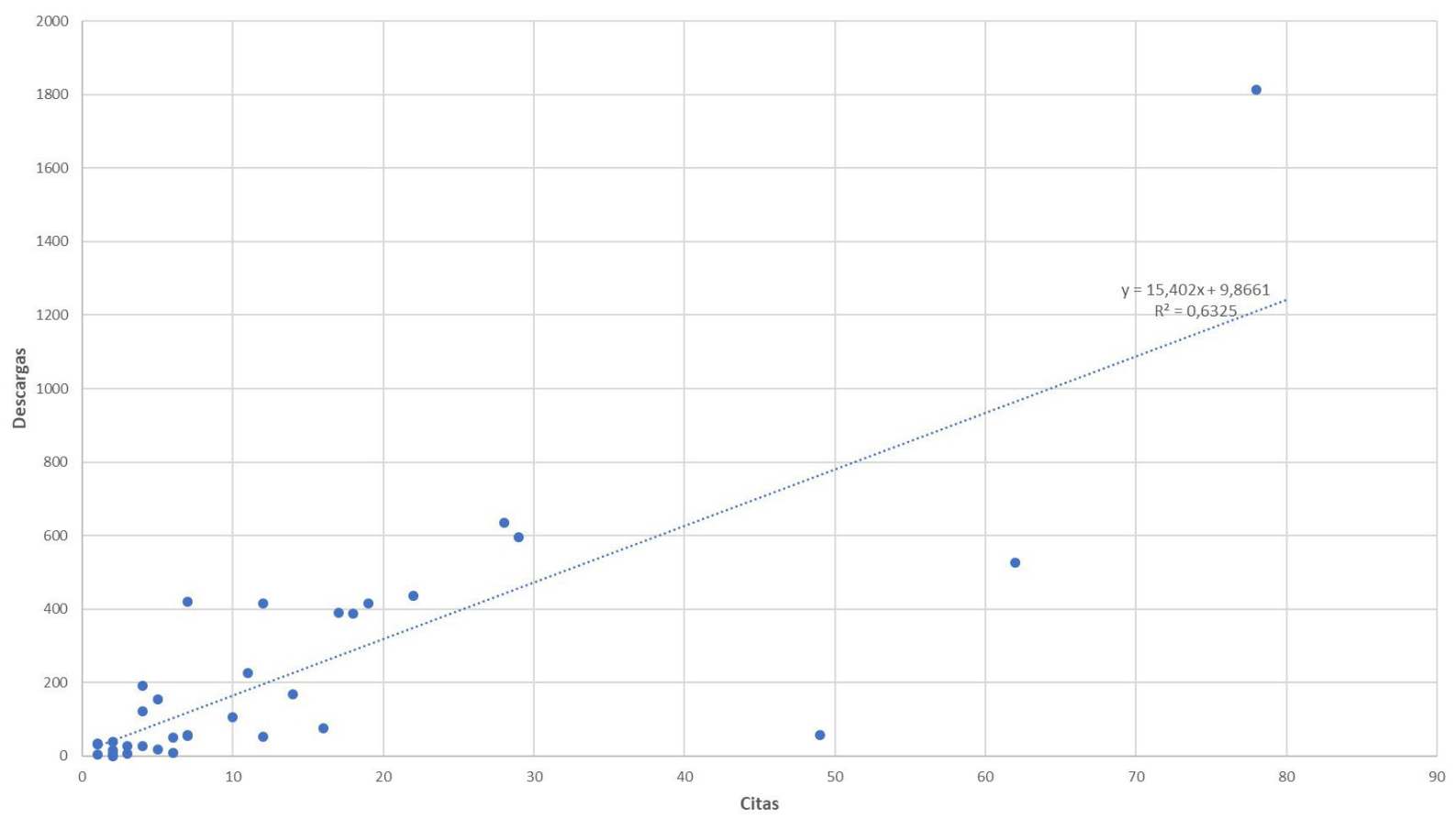

Se aprecia que el uso de los títulos tanto para su lectura como para su referencia en la producción científica no es regular. Las revistas que muestran un uso y una utilidad en todas las anualidades son algunas de las revistas más reputadas que ya hemos señalado con anterioridad, concretamente: JASIST, Online Information Review, Journal of Documentation e Information Processing \& Management.

A nivel agregado por años, se ha podido comprobar que existe una fuerte correlación lineal $(r=0,79)$ entre el número de citas que reciben las revistas suscritas y el número de descargas de esas revistas el año anterior a la publicación de los documentos. Tal y como se aprecia en la Figura 5 , en general a medida que aumenta una variable aumenta la otra, aunque hay alguna revista que no se ajusta muy bien al modelo y presenta una cierta descompensación entre el número de citas y el de descargas.

\section{DISCUSIÓN}

Los resultados obtenidos en este trabajo deben valorarse teniendo en cuenta que este análisis parte de la producción bibliográfica en revistas de una categoría concreta de Scopus y, por tanto, hay que considerar de inicio las limitaciones de cobertura y de consistencia de la mencionada clasificación de Scopus. Como han señalado Urbano y Ardanuy (2020) en un análisis de la producción científica de cuatro países europeos, las revistas englobadas en la categoría LIS no pertenecen únicamente al ámbito de la Documentación. De hecho, el $72 \%$ de los títulos de dicha categoría se adscriben a dos o más categorías, y casi el $20 \%$ a cuatro o más. De ahí que cubran campos vinculados no solo a otras Ciencias Sociales sino también a Humanidades, Ciencias Experimentales y Ciencias Tecnológicas. Por otro lado, creemos necesario poner de relieve la dificultad intrínseca de análisis como el presente por el tiempo que se necesita invertir en la limpieza y normalización manual de datos. Martin y otros (2016) destacan esta circunstancia: "considerando el tiempo y el esfuerzo requerido para recuperar y limpiar datos de referencias (...), es necesario preguntarse si el valor de estos datos para tomar decisiones sobre las colecciones supera el valor del tiempo que el personal necesita para su desarroIlo". En esta investigación, esas dificultades se pueden clasificar en los siguientes puntos:

- Adscripción. La revisión manual de los registros descargados de Scopus permitió identificar artículos atribuidos erróneamente a la Universidad de Salamanca o firmados por alumnos de doctorado cuya filiación era otra institución. Asimismo, se identificó un artículo con dos registros idénticos asociados. Consideramos que este dato es revelador de las limitaciones de Scopus a la hora de analizar la producción científica de una institución. 
- Normalización de los autores y filiación. Fue necesario acometer la normalización de los nombres de los autores y su filiación antes de clasificarlos por áreas temáticas. Algunos autores se limitaban a indicar su universidad, otros añadían la facultad, el departamento, o el instituto de investigación y había quienes ofrecían varias adscripciones. Los clasificamos atendiendo al área de conocimiento al que pertenecían o en su defecto a su departamento, en el caso de los académicos. A los profesionales les ubicamos en el servicio de la institución o empresa a la que pertenecían. Las dificultades para asignar consistentemente filiaciones a los autores han sido puestas de relieve en otros estudios (Shu y otros, 2018).

- Referencias bibliográficas. El campo "referencias" de Scopus presenta numerosos errores de codificación y normalización, lo que nos obligó a emplear un considerable tiempo en extraer y normalizar los títulos de revistas (títulos abreviados, desarrollados, con o sin artículo, etc.).

- Datos de descargas. Las estadísticas de descargas que los suministradores entregan a las bibliotecas presentan anomalías que obligan a quien las utiliza a tratar de aclarar algunos datos previamente a iniciar su análisis. Esta circunstancia ha sido puesta de relieve por Bucknell (2012) y constatada en estudios recientes (Fernández-Ramos y otros, 2019).

Centrándonos en los resultados de esta investigación y en la interpretación que se puede hacer de los mismos, cabe destacar que, desde el punto de vista de la producción científica, se ha observado un claro dominio de la procedente de la Universidad de Salamanca. Los datos guardan relación con la existencia en ella de un área de conocimiento de Biblioteconomía y Documentación bien nutrida, que en ocasiones publica en coautoría con alumnos de máster y de doctorado. También es destacable la colaboración con bibliotecarios, poco habitual en la Universidad de León. Conviene indicar, igualmente, que en las Universidades de Salamanca y Valladolid se imparten estudios de Comunicación $y$, como se ha constatado en este estudio, los académicos de este ámbito juegan un papel relevante en la publicación en revistas de la categoría LIS de Scopus. De hecho, entre los sectores académicos de Informática y de Comunicación son responsables del $30 \%$ de los documentos publicados en esta categoría, por encima del $27 \%$ que se adscribe al área de la Documentación.

La categoría LIS de Scopus es liderada por autores de Informática, Empresa y Sistema de Información, como constatan Urbano y Ardanuy (2020).
También Chang (2019) confirma, tomando como referencia las revistas de Documentación en JCR, que solo el $33,3 \%$ de las revistas publican más de la mitad de sus artículos gracias a autores del ámbito de la Documentación. Urbano y Ardanuy (2020) encuentran que los investigadores de Documentación representan en torno al $30 \%$ en el total de la producción que analizan, algo más -33,74\%si solo se consideran los artículos. En esta misma línea, Chang (2018) halla que autores externos a Documentación contribuyen con el $46,5 \%$ de los artículos. El posicionamiento de los autores de Documentación es débil en estos estudios y, con todo, ligeramente superior al que hemos observado en este trabajo.

Nuestro estudio muestra que entre las fuentes de publicación destaca la revista El profesional de la información, en la que se han difundido 36 artículos del total, y que en los últimos años es conocida como medio para publicar contenidos de Comunicación además de los de Documentación. En este sentido, Ollé y otros (2016) constatan que los editores de revistas de Documentación han buscado recientemente expandirse hacia áreas relacionadas: Comunicación (32\%), Gestión (25\%) e Informática (19\%). Aharony (2012) tras analizar diez revistas del ramo confirma la apertura de las revistas de LIS a otros campos y que la Documentación solo era el ámbito del 36,36\% de los autores. Las políticas editoriales de estas publicaciones y su apertura temática son agentes activos determinantes de la investigación y convierten a las revistas en algo más que en meros vehículos de difusión de información.

Respecto a la productividad de los autores de nuestro estudio, esta se ajusta a los enunciados formulados por Lotka, en los que establece que una mayoría de los autores escribe muy pocos artículos y una minoría son muy prolíficos y publican la mayor parte de la literatura científica sobre un tema. De hecho, el $89 \%$ de los 282 autores identificados solo han firmado uno o dos documentos. Además, se ha observado una marcada tendencia a la coautoría (el 86,2 \% de los artículos), con un notable carácter intradisciplinar en dicha colaboración. Por su parte, Chang (2018) indica que la colaboración interdisciplinar es escasa pero constante en el ámbito de la Documentación. Urbano y Ardanuy (2020) señalan que la colaboración con otras disciplinas es muy limitada, solo el $21,5 \%$ de los artículos se realizan en colaboración con otras ciencias, y que esta se da principalmente con investigadores de Comunicación y con profesionales de la información. En nuestro estudio la colaboración más frecuente ha sido entre el ámbito de la Documentación y el de Informática, constatando 
la influencia creciente de esta en la Documentación ya señalada hace casi dos décadas por Tang (2004). La colaboración con bibliotecarios ha sido constatada también en este trabajo suponiendo el $13,77 \%$ del total. Se demuestra que la forma en que se organiza y estructura la docencia en las universidades influye en la colaboración que se da después en las investigaciones y que la creación de grupos también resulta condicionada. No se observa en este estudio, sin embargo, la existencia de equipos pluridisciplinares consolidados con el epicentro en el sector de la Documentación, más bien diversas áreas que comparten la utilización de las revistas de la categoría LIS de Scopus.

En cuanto a las pautas de citación, la media de citas por artículo es elevada, algo que coincide plenamente con lo observado en estudios similares recientes enfocados en el análisis de citación de revistas concretas de Documentación (Singh y Ravikumar, 2016; Devi y otros, 2018). En esa misma línea, Belter y Kashe (2016) constatan la necesidad de disponer de muchos artículos para la investigación, circunstancia que se demuestra en que el número de referencias por artículo se ha ido incrementando en los años cubiertos por su estudio (2009-2013). Los mismos autores afirman que es posible identificar un núcleo de revistas más citadas, circunstancia confirmada en este estudio en el que se han aislado las revistas que han recibido diez o más citas y dónde se aprecia una distancia clara entre las referencias recibidas por El profesional de la información, JASIST y Scientometrics y las restantes revistas. Las revistas que no figuran en la tabla II podrían considerarse periféricas porque se referencian con menos intensidad.

Según Shu y otros (2018), los investigadores citan solo una fracción de las revistas suscritas por sus bibliotecas y esa fracción estaría decreciendo, lo que haría perder valor a los paquetes de revistas contratados. Esta circunstancia no es corroborada, sin embargo, en este análisis en el ámbito de la Documentación. Las revistas de los principales editores integradas en los big deals tienen una presencia relevante entre los títulos más citados. El $42,69 \%$ de las citas provienen de revistas suscritas en los proveedores analizados.

En el global de las citas se confirma la preferencia por los contenidos de Elsevier, situación común constatada en otros estudios que analizan descargas (Fernández-Ramos y otros, 2019). Sin embargo, si atendemos a las revistas adscritas a la categoría LIS, cabe apuntar que son los títulos de Emerald los que recaban un número superior de citas en un abanico amplio de títulos. Es preciso señalar que este suministrador es el que distri- buye un número mayor de títulos con contenidos propiamente de Documentación. Esta situación difiere de los resultados encontrados por Currie y Monroe-Gulick (2013) referidos al ámbito general de las Ciencias Sociales. En el estudio referido los principales editores citados fueron Elsevier y Wiley-Blackwell. Esta circunstancia apunta a que las generalizaciones han de realizarse con precaución, incluso entre disciplinas próximas.

El análisis de las revistas citadas presentes en Scopus confirma la preferencia por citar contenidos de Documentación, de Informática y de Comunicación. En este sentido, Chen y otros (2018) afirman que los autores de Documentación consiguen $30 \%$ de sus referencias de campos externos, mientras que Levitt y Thelwall (2009) argumentan que las frecuentes referencias a otras disciplinas explican el mayor impacto de sus trabajos.

Se constata que son las Universidades de León y Salamanca las que predominantemente leen y citan las revistas de Documentación, situación acorde con la existencia en ellas de académicos del área de Biblioteconomía y Documentación. Se aprecian, no obstante, diferencias en las preferencias de los investigadores de ambas universidades. En este sentido, Belter y Kashe (2016) subrayan que, aunque exista un amplio acuerdo entre instituciones sobre las revistas más usadas en una disciplina, pueden observarse diferencias en función de intereses locales y prioridades investigadoras. No siempre las revistas más citadas en toda una disciplina (JCR) son las más referenciadas en una institución en particular.

Para tratar de conocer la correspondencia entre la utilización de revistas y su utilidad en la producción científica por medio de las citas hemos optado por relacionar las citas de cada revista en cada una de las anualidades (2011-2017) con las descargas efectuadas un año antes. Somos conscientes de que el método empleado solo permite una aproximación a la realidad pues, como hemos puesto de relieve en la metodología, la fecha de descarga de un artículo citado es incierta, aunque mayoritariamente sea próxima a la fecha de citación. Asimismo, no se puede obviar que no todas las descargas tienen como finalidad la investigación, sino que pueden ser realizadas con objetivos de aprendizaje por parte de los profesores o de los alumnos. Finalmente, como ya afirmó Nicholas (2008), no existen evidencias al respecto que indiquen que todos los documentos descargados se lleguen a leer y por tanto puedan ser utilizados para construir investigación.

En cualquier caso, se ha comprobado que existe un paralelismo claro entre citas y descargas ya 
que se constata que generalmente las revistas descargadas se citan y el número de descargas es superior al de referencias. Como han apuntado Martin y otros (2016) ambas métricas no se pueden comparar. Descargar un artículo requiere menos esfuerzo que citar un artículo. Por tanto, la situación habitual es que el número de descargas de un título muy utilizado sea muy superior que el número de citas de un artículo muy citado. Como algunos estudios anteriores han confirmado (Tsay, 1998; Blecic, 1999; McDonald, 2007) el uso de las revistas es una variable significativa para predecir patrones de citas. En este trabajo se ha comprobado estadísticamente que existe una correlación significativa entre ambas métricas.

\section{CONCLUSIONES}

Una de las principales conclusiones de este trabajo es la confirmación de la importancia que aún tienen las revistas científicas como vehículo fundamental en la transmisión del conocimiento, atestiguado por el hecho de que más de la mitad de las referencias analizadas en este estudio se correspondieran con esta tipología documental. Esta vigencia de las revistas científicas ha sido puesta de relieve recientemente por Kim y otros (2019) y por Herman y otros (2020). Estos últimos señalan que las revistas son el único producto que cumple todavía consistentemente con todas las funciones que tradicionalmente se les han atribuido -registro, curación, evaluación, distribución y archivo- y que son necesarias para institucionalizar y añadir con confianza una contribución académica al conjunto del conocimiento.

La trazabilidad digital de los resultados de la investigación es especialmente relevante en tiempos de una creciente demanda de rendición de cuentas en el uso de fondos públicos. La transformación digital acaecida en los últimos años ha permitido que los datos registrados en múltiples plataformas tengan una potencial repercusión en el modo en que la investigación y actividades relacionadas pueden ser evaluadas y recompensadas. Junto a las métricas asentadas, basadas en el posicionamiento de las revistas en las principales bases de datos bibliográficas o en las citas recibidas, han ido surgiendo otras métricas alternativas, entre las que se encuentran los datos de descargas. Estas métricas alternativas están en proceso de consolidación, principalmente porque requieren ser estudiadas en relación con las métricas tradicionales. El presente estudio ha permitido dar un paso en esa línea al confirmar que existe una correlación significativa entre citas y descargas. Las descargas señalan la utilización de unos contenidos y las citas la utilidad de esos mismos contenidos para construir nueva investigación.
Sin querer entrar a valorar aquí la conveniencia de mantener o no las suscripciones a los big deals en estos momentos en que proliferan vías alternativas de acceso a los contenidos y documentos en acceso abierto, las citas a revistas contratadas por medio de paquetes confirman que, al menos en el caso de la Documentación, estos editores distribuyen contenidos de calidad -útiles-y facilitan su acceso. De esta forma dotan de visibilidad a las revistas que distribuyen y promueven su lectura y posterior citación. En nuestro estudio se ha observado que, además de los contenidos suscritos, se han citado de forma considerable revistas distribuidas por otros medios o en acceso abierto, muchas de ellas españolas o internacionales bien posicionadas en los rankings. No obstante, es preciso señalar que las revistas españolas han sido más utilizadas para publicar que para citar, algo que consideramos atribuible a que la accesibilidad lingüística favorece la publicación en ellas. Sin embargo, la indización en las bases de datos de citas, Web of Science y Scopus, son factores determinantes en la selección de documentos que se leen y se citan. La presión por publicar en revistas bien posicionadas que sufren los investigadores les conduce a utilizar en sus artículos contenidos de revistas reputadas.

Otra conclusión remarcable es que las preferencias de los investigadores de las cuatro universidades analizadas no son estrictamente homogéneas, sino que están condicionadas en gran medida por sus diferentes líneas de investigación, que lógicamente varían entre diferentes disciplinas, pero que incluso se aprecian dentro de la misma área de conocimiento. Además, se ha constatado la convivencia de autores de disciplinas variadas en los artículos publicados en las revistas de la categoría LIS, así como una escasa colaboración entre disciplinas que hace cuestionar la creencia tradicional de que la Documentación es un campo científico interdisciplinar, como ya señalaron Urbano y Ardanuy (2020).

Consideramos que, dado que los investigadores del ámbito de la Documentación no protagonizan más allá del $30 \%$ de los contenidos insertos en las revistas de la categoría LIS, sería conveniente, en futuros estudios, indagar en su presencia en las revistas de otras categorías que pueden resultar afines como serían las de Ciencias Sociales, Humanidades o Informática. Asimismo, otra línea de investigación que nos planteamos es extender el estudio de la relación entre descargas y citas a otras categorías científicas y tratar de ampliar el ámbito geográfico. Ambas actuaciones con el fin de establecer paralelismos y diferencias de comportamiento en la lectura y citación 
entre investigadores y confirmar la relación entre ambas métricas. Para el desarrollo de estudios de mayor amplitud la opción de automatizar este proceso de normalización y filtrado de los datos sería algo deseable, pero muy costoso en términos de tiempo y recursos mientras desde los agentes implicados (las bases de datos, las propias revistas, e incluso los autores) no se emprenda con decisión la labor de normalizar la información bibliográfica.

\section{AGRADECIMIENTOS}

Esta investigación ha sido financiada por el Programa Estatal de Investigación, Desarrollo e Innovación Orientada a los Retos de la Sociedad 2017, convocada por el Ministerio de Economía, Industria y Competitividad y la Agencia Estatal de Investigación (CSO2017-87956-R) y por el programa de subvenciones destinadas al apoyo de los grupos de investigación reconocidos de universidades públicas de Castilla y León iniciados en 2018, convocada por la Consejería de Educación de la Junta de Castilla y León (LE028G18).

\section{ACKNOWLEDGEMENTS}

This research has been funded by the State Program for Research, Development and Innovation Oriented to the Challenges of Society 2017, convened by the Ministerio de Economía, Industria y Competitividad and the Agencia Estatal de Investigación (CSO2017-87956-R) and by the grant program aimed at supporting recognized research groups of public universities in Castilla y León started in 2018, convened by the Consejería de Educación de la Junta de Castilla y León (LE028G18).

\section{REFERENCIAS}

Aharony, N. (2012). Library and information science research areas: A content analysis of articles from the top 10 journals 2007-2008. Journal of Librarianship and Information Science, 44 (1), 27-35. https://doi. org/10.1177/0961000611424819

Belter, Ch.W., y Kaske, N. K. (2016). Using Bibliometrics to Demonstrate the Value of Library Journal Collections. College \& Research Libraries, 77 (4), 410-422. https://doi.org/10.5860/crl.77.4.410

Blecic, D. D. (1999). Measurements of Journal Use: an analysis of the correlations between three methods. $\mathrm{Bu}$ lletin of the Medical Library Association, 87 (1), 20-25.

Bucknell, T. (2012). Garbage in, gospel out: twelve reasons why librarians should not accept cost-per-download figures at face value. The Serials Librarian, 63 (2), 192212. https://doi.org/10.1080/0361526X.2012.680687

Chambers, G. R., y Healey, J. S. (1973). Journal citations in master's Theses. One Measurement of a Journal Collection. Journal of the American Society for
Information Science, 24 (5), 397-401. https://doi. org/10.1002/asi.4630240511

Chang, Y. M. (2018). Examining interdisciplinarity of library and information science (LIS) based on LIS articles contributed by non-LIS authors. Scientometrics, 116 (3), 15891613. https://doi.org/10.1007/s11192-018-2822-7

Chang, Y. M. (2019). Are articles in library and information science (LIS) journals primarily contributed to by LIS authors? Scientometrics, 121, 81-104. https://doi. org/10.1007/s11192-019-03186-w

Chen, C., Li, Q., Deng, Z., Chiu, K., y Wang, P. (2018). The preferences of Chinese LIS journal articles in citing works outside the discipline. Journal of Documentation, 74 (1), 99-118. https://doi.org/10.1108/JD-04-2017-0057

CIBER. (2009). E-Journals: their use, value and impact: a Research Information Network report. London: City University. http://www.rin.ac.uk/system/files/attachments/E-journals-report.pdf. report.pdf [Fecha de consulta: 30/05/2020]

Connaway, L. S., Dickey, T.J., y Radford, M. L. (2011). "If it is too inconvenient, I'm not going after it:". Convenience as a critical factor in information-seeking behaviors. Library \& Information Science Research, 33 (3), 179-190. https://doi.org/10.1016/j.lisr.2010.12.002

Currie, L., y Monroe-Gulick, A. (2013). What do our faculty use? An interdisciplinary citation analysis study. The Journal of Academic Librarianship, 39 (6), 471-480. https://doi.org/10.1016/j.acalib.2013.08.016

Devi, J., Kumar, D., Jangra, y R. (2018). Citation Trends in Library \& Information Science: A Bibliometric Study of 'Library Trends' from 2012 to 2016. En: Changing Digital Landscape in SMART Environment NCCDLSE 2018, 283-291. Gurugram: Ansal University.

Fernández-Ramos, A., Rodríguez-Bravo, B., Alvite-Díez, M. L., Santos-De-Paz, L., Morán-Suárez, M. A., Gallego-Lorenzo, J., y Olea, I. (2019). Evolution of the big deals use in the public universities of the Castile and Leon region, Spain. El Profesional de la Información, 28 (6), e280519. https://doi.org/10.3145/epi.2019.nov.19

González-Solar, L., y Fernández-Marcial, V. (2019). SciHub, a challenge for academic and research libraries. El Profesional de la Información, 28 (1), e280112. https://doi.org/10.3145/epi.2019.ene.12

Herman, E., Akeroyd, J., Bequet, G., Nicholas, D., y Watkinson, A. (2020). The changed -and changing-landscape of serials publishing: review of the literature on emerging models. Learned Publishing 33 (3), 213-229. https://doi.org/10.1002/leap.1288

Himmelstein, D. S., Rodríguez-Romero, y A., Levernier, J. G., Munro, T.A., McLaughlin, S. R., Tzovaras, B. G., y Greene, C. (2018). Sci-Hub provides access to nearly all scholarly literature. eLife, 1 (7), e32822. https:// doi.org/10.7554/eLife. 32822

Hoffmann, K., y Doucette, L. (2012). A Review of citation Analysis Methodologies for Collection Management. College \& Research Libraries, 73 (4), 321-335. https:// doi.org/10.5860/crl-254

Ivanov, A. O., Johnson, C. A., y Cassady, S. (2020). Unbundling practice: the unbundling of big deal journal packages as an information practice. Journal of Documentation 76 (5), 1051-1067. https://doi. org/10.1108/JD-09-2019-0187 
Kim, L: Portenoy, J. H., West, J. D., y Stovel, K. W. (2019). Scientific Journals Still Matter in the Era of Academic Search Engines and Preprint Archives. Journal of the Association for Information Science and Technology, 1- 9. https://doi.org/10.1002/asi.24326

Kurtz, M. J., y Bollen, J. (2010). Usage Bibliometrics. Annual Review of Information Science and Technology, 44, 3-64. https://doi.org/10.1002/aris.2010.1440440108

Levitt, J.M, y Thelwall, M. (2009). Citation levels and collaboration within library and information science. Journal of the Association for Information Science and Technology, 60 (3), 434-442. https://doi.org/10.1002/ asi. 21000

Luther, J. (2002). White paper on Electronic Journal Usage Statistics. Whatsington DC: Council on Library an Information Resources.

Martin, V., Gray, T., Kilb, M., y Minchew, T. (2016). Analyzing Consortial "Big Deals" via a Cost-Per-Cited-Reference (CPCR) Metric. Serials Review, 42 (4), 293-305. https://doi.org/10.1080/00987913.2016.1248218

McDonald, J. D. (2007). Understanding Journal Usage: a statistical Analysis of Citation and Use. Journal of the American Society for Information Science and Technology, 58 (1), 39-50. https://doi.org/10.1002/asi.20420

Medeiros, N. (2007). Uses of necessity or uses of convenience? What usage statistics reveal and conceal about electronic serials. En: D. C. Fowler (ed.). Usage statistics of e-serials. Boca Raton, Fl.: CRC Press. P. 233-243.

Nicholas, D. (2008). If we do not understand our users, we will certainly fail. En: The E-Resources Management Handbook 1, United Kingdom: Serials Group. Disponible en: https://www.uksg.org/sites/uksg.org/ files/21-Nicholas-E2Q]FQYMRRMG3RQD.pdf [Fecha de consulta: 30/05/2020]

Nicholas, D., Boukacem-Zeghmouri, C., Xu, Jie, Herman, E., Clark, D., Abrizah, A., Rodriguez-Bravo, B., y Świgoń, M. (2019). Sci-Hub: The new and ultimate disruptor? View from the front. Learned Publishing, 32 (2), 147-153. https://doi.org/10.1002/leap.1206

Nicholas, D., Jamali, H. R., Herman, E., Watkinson, A., Abrizah, A., Rodríguez-Bravo, B., Boukacem-Zeghmouri, C., Xu, J., Swigon, M., y Polezhaeva, T. (2020). A global questionnaire survey of the scholarly communication attitudes and behaviours of early career researchers. Learned Publishing, 33, https://doi. org/10.1002/leap.1286

Ollé, C., López-Borrull, A., y Abadal, E. (2016). The challenges facing library and information science journals: Editor's opinions. Learned Publishing, 29 (2), 89-94. https://doi.org/10.1002/leap.1016

Rodríguez-Bravo, B., y Alvite-Díez, M. L. (2006a). The Use of Electronic Journals in Academic Libraries in Castilla y León. En: Libraries Without Walls 6: Evaluating the Distributed Delivery of Library Services. London: Facet, pp. 125-137.

Rodríguez-Bravo, B., y Alvite-Díez, M. L. (2006b). Uso de las revistas-e suministradas por Emerald en bibliotecas universitarias españolas (2002-2005). El Profesional de la Información. 15 (6), 464 - 472. http:// profesionaldelainformacion.com/contenidos/2006/noviembre/08.pdf
Rodríguez-Bravo, B., y Alvite-Díez, M. L. (2011). An analysis of the use of electronic journals in an academic context: Developments and profitability. Serials Review, 37 (3), 118-195. https://doi.org/10.1016/j. serrev.2011.06.003

Rodríguez-Bravo, B., y Alvite-Díez, M. L. (2013). Impact of the consumption of electronic contents on research productivity in the universities of Castile and Leon. Library Collections, Acquisitions, and Technical Services, 37 (3-4), 85-106. https://doi.org/10.1016/J. LCATS.2013.09.007

Rodríguez-Bravo, B., Alvite-Díez y M. L., Barrionuevo-Almuzara, L. (2012a). Trends and models in the consumption of electronic contents. An analysis of the journals most widely used in Spanish universities. Journal of Academic Librarianship, 38 (2), 42-59. https://doi.org/10.1016/j.acalib.2011.11.007

Rodríguez-Bravo, B., Alvite-Díez, M. L., Morán-Suárez, M. A. y Barrionuevo-Almuzara, L. (2008). Patterns of use of electronic journals in Spanish university libraries. Serials Review, 34 (2), 115-128. https://doi.or$\mathrm{g} / 10.1016 / \mathrm{j}$. serrev.2008.01.002

Rodríguez-Bravo, B., Alvite-Díez, M. L., Morán-Suárez, M. A., y Marraud, G. (2012b). Impacto de la contratación de colecciones de revistas electrónicas en la productividad de la Universidad de Vigo. El Profesional de la Información, 21 (6), 585-594. https://doi.org/10.3145/epi.2012. nov.05

Salisbury, L., y Smith, J. S. (2010). The use of Web of Knowledge to study publishing and citation use for local researchers at the campus level. Collection Management, 35 (2), 69-82. https://doi.org/10.1080/01462671003597959

Sanville, T. J. (2001). A method out of the madness: OhioLink's collaborative response to the serial crisis: four years later progress report. Serials, 14 (2), 163177. https://doi.org/10.1629/14163

Shu, F., Mongeon, P., Haustein, S., Siler, K., Alperin, J. P., y Larivière, V. (2018). Is it such a big deal? On the cost of journal use in the digital era. College \& Research Libraries, 79 (6), 785-798. https://doi.org/10.5860/crl.79.6.785

Singh, S., y Ravikumar, S. (2016). Citation Concentration in ASLIB Proceedings Journal: A Comparative Study of 2005 and 2015 Volumes. Disponible en: https://ir.inflibnet.ac.in/bitstream/1944/2036/1/32.pdf [Fecha de consulta: 30/05/2020]

Tang, R. (2004). Evolution of the interdisciplinary characteristics of information and library science. Proceedings of the American Society for Information Science and Technology, 41 (1), 54-63. https://doi.org/10.1002/ meet. 1450410107

Tenopir, C., Allard, S., Bates, B. J., Levine, K. J., King, D. W., Birch, B., y Caldwell, C. (2011). Perceived value of scholarly articles. Learned Publishing, 24 (2), 123132. https://doi.org/10.1087/20110207

Tsay, M. Y. (1998). The relationship between journal use in a medical library and citation use. Bulletin of the Medical Library Association, 86 (1), 31-39.

Urbano, C., Anglada, L. M., Borrego, Á., Cantos, C., Cosculluela, A., y Comellas, N. (2004). The use of consortially purchased electronic journals by the CBUC (2000-2003). D-Lib Magazine, 10 (6). http:// www. dlib.org/dlib/june04/anglada/06anglada.html 
Urbano, C., y Ardanuy, J. (2020). Cross-disciplinary collaboration versus coexistence in LIS serials: analysisi of authorship affiliations in four European countries. Scientometrics. https://doi.org/10.1007/s11192-020-03471-z

White, P. (2019). Using Data Mining for Citation Analysis. College \& Research Libraries, 80 (1), 76-93. https:// doi.org/10.5860/crl.80.1.76
Wilson, C. S., y Tenopir, C. (2008). Local citation analysis, publishing, and reading patterns: using multiple methods to evaluate faculty use of an academic library's research collection. Journal of the American Society for information Science and Technology, 59 (9), 13931408. https://doi.org/10.1002/asi.20812 